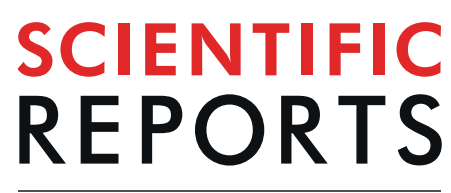

natureresearch

Check for updates

\title{
Expression of a Barhl1a reporter in subsets of retinal ganglion cells and commissural neurons of the developing zebrafish brain
}

\author{
Shahad Albadri ${ }^{1,3}$, Olivier Armant ${ }^{2}$, Tairi Aljand-Geschwill ${ }^{1}$, Filippo Del Bene ${ }^{3}$, Matthias Carl ${ }^{4}$, \\ Uwe Strähle ${ }^{2}$ \& Lucia Poggi ${ }^{1,4} \bowtie$
}

Promoting the regeneration or survival of retinal ganglion cells (RGCs) is one focus of regenerative medicine. Homeobox Barhl transcription factors might be instrumental in these processes. In mammals, only barhl2 is expressed in the retina and is required for both subtype identity acquisition of amacrine cells and for the survival of RGCs downstream of Atoh7, a transcription factor necessary for RGC genesis. The underlying mechanisms of this dual role of Barhl 2 in mammals have remained elusive. Whole genome duplication in the teleost lineage generated the barhl1 $a$ and barhl2 paralogues. In the Zebrafish retina, Barhl2 functions as a determinant of subsets of amacrine cells lineally related to RGCs independently of Atoh7. In contrast, barhl1a expression depends on Atoh7 but its expression dynamics and function have not been studied. Here we describe for the first time a Barhl1a reporter line in vivo showing that barhl1a turns on exclusively in subsets of RGCs and their post-mitotic precursors. We also show transient expression of barhl1a:GFP in diencephalic neurons extending their axonal projections as part of the post-optic commissure, at the time of optic chiasm formation. This work sets the ground for future studies on RGC subtype identity, axonal projections and genetic specification of Barhl1a-positive RGCs and commissural neurons.

BarH-like homeodomain (BARHL) transcription factors play crucial roles in the control of neural cell fate specification, migration, subtype identity acquisition and survival during development of the retina and the brain ${ }^{1-7}$. Studies have also implicated Barhl in neurodegenerative and neoplastic disorders ${ }^{8,9}$. Previous barhl studies have suggested that whole genome duplication (WGD) during vertebrate evolution generated two homologs of barhl: barhl1 and barhl210. In addition, studies in Xenopus and mouse suggest that barhl2 (previously named $M B H 1$ and $X B H 1$ ) is the only family member expressed in the retina of tetrapods ${ }^{11,12}$. Specifically, barhl2 expression is found in both amacrine cells and retinal ganglion cells (RGCs) of the developing and mature retina ${ }^{13,14}$. Furthermore, Barhl2 is both sufficient and essential for determining the subtype specific identity of amacrine cells as well as to promote the maturation and survival of RGCs. Lastly, the expression of barhl2 in RGCs appears to depend on Atoh7 (also known as Ath5) - a bHLH transcription factor required for the specification of RGCs in vertebrates $^{13-19}$.

In Zebrafish, three barhl paralogs were found ${ }^{4,7,10}$. Studies based on both Barhl protein sequence analysis and conserved gene synteny between barhl genes suggest that they likely arise from the additional round of WGD in teleosts followed by the loss of one barhl paralog ${ }^{10}$. These studies also have revealed that barhllb (previously called barhl1 or barhl1.1) and barhl1a (previously called barhl1.2) are more closely related to the mammalian barhl1. Conversely, the barhl2 paralogue is more closely related to the mammalian barhl2 ${ }^{10}$. Concordantly, similarly to its barhl1 counterpart in tetrapods, barhl1b lost its retinal expression, maybe due to a redundant function with barhl1 $a$ and relaxed evolutionary pressure in its locus ${ }^{4,10}$. On the contrary, retinal expression of barhl1a and barhl2 is retained, but these two paralogs appear to have diversified their function in retinal lineages ${ }^{10}$. In favour of this hypothesis, studies have shown that, similarly to the mammalian counterpart, Barhl2 is an amacrine cell

${ }^{1}$ Centre for Organismal Studies, University of Heidelberg, Heidelberg, Germany. ${ }^{2}$ Institute of Biological and Chemical Systems, Biological Information Processing Karlsruhe Institute of Technology, Eggenstein-Leopoldshafen, Germany. ${ }^{3}$ Institut de la Vision, Sorbonne Université, INSERM, CNRS, Paris, France. ${ }^{4}$ Department of Cellular, Computational

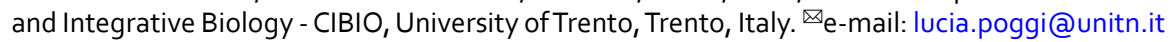


subtype identity-biasing factor downstream of the transcription factor Ptfla $\mathrm{a}^{20}$. Furthermore, taking advantage of the zebrafish transgenesis combined with accessibility to 3D time-lapse imaging ${ }^{21-25}$ we have previously shown that barhl2-expressing amacrine subtypes consistently arise within the lineage of Atoh7 upon reproducible asymmetrical divisions of RGC progenitors ${ }^{20}$ (see also Fig. 9). Interestingly, while barhl2 turns on exclusively in amacrine cells under the control of Ptfla ${ }^{20}$, the expression of barhl1a depends on Atoh7 and appears therefore to be restricted to the ganglion cell layer $(\mathrm{GCL})^{10}$. The generation pattern of individual barhlla-expressing cells within the Atoh7 cell lineage, the lineage relationship among barhlla and barhl2 -expressing cells in vivo as well as the role played by Barhlla in RGC genesis have so far remained unknown.

To address these questions, direct and dynamic visualization of barhlla-expressing cells is needed. We used Tol2 transposase-mediated BAC transgenesis to generate a Barhl1a reporter line where GFP expression is driven by barhl1 a regulatory regions. This reporter recapitulates barhl1 a mRNA expression and helped us determine that barhl1 $a$ turns on exclusively in a sub-population of RGCs after the cell cycle exit of atoh7-expressing progenitors. In addition, the reporter allows us to visualize axonal connectivity derived from barhl1a-expressing neurons. Furthermore, we provide the first description of the expression dynamic of barhl1a in commissural neurons of the forebrain. This study thereby provides the foundation for further investigations of the role of Barhlla transcription factors in nerve cell subtype identity acquisition and maintenance in this in vivo model system.

\section{Results}

Barhl1a in the retina is up-regulated in a subset of atoh7-expressing post-mitotic RGC precursors. In order to visualize barhlla-expressing cells in vivo we generated a $\mathrm{Tg}$ (barhl1a:GFP) transgenic line expressing the reporter eGFP under barhlla regulatory genomic elements. To this end, a BAC spanning the barhlla genomic locus was used to perform Tol2 transposon-mediated BAC transgenesis replacing eGFP with the barhlla coding sequence (see Methods). The newly generated $\mathrm{Tg}$ (barhl1a:GFP) transgenic embryos displayed the distribution pattern of GFP as expected from barhlla expression in the posterior thalamus and zona limitans intratalamica $(\mathrm{ZLI})^{4}$ (Supplementary Fig. 1A). To further assess the reliability of the Tg(barhl1a:GFP) line, we compared the spatial expression pattern of barhl1 $a$ mRNA and GFP mRNA in $T g$ (barhlla:GFP) embryos by double fluorescent in situ hybridization (FISH). Transcripts of both genes can be consistently found as shown, for example, at 35 and 40 hours post-fertilization (hpf), in the developing GCL as well as in the optic tectum (Fig. 1).

To directly investigate the dynamics of the appearance of individual Barhl1a:GFP-positive cells in the retina, we used 3D time-lapse imaging on $\mathrm{Tg}$ (barhl1a:GFP) embryos. Imaging of the developing retina of $\mathrm{Tg}$ (barhl1a:GFP) embryos for at least 20 hours revealed that GFP positive cells become first visible in the ventral retina at $\sim 29-30 \mathrm{hpf}$ and spread subsequently to the nasal, dorsal and temporal retina in a temporal and spatial fashion predictive of the wave of RGC differentiation (Fig. 2A-D and Supplementary Movie 1) ${ }^{26}$. Individual cells turning on barhl1a:GFP can be seen located either in the basal half or in the apical half of the neuroepithelium, from where they subsequently migrate basally until they reach the GCL (Fig. 2A-D and Supplementary Movie 1). We never observed Barhlla:GFP cells migrating to the apical surface to undergo division. This suggests that barhl1a:GFP turns on in RGC precursors after the terminal division of atoh7-expressing neuroepithelial cells. Immuno-labelling of Tg(barhlla:GFP) embryos with an antibody to Cxcr4b, a marker of post-mitotic RGCs ${ }^{27}$ further supports our notion that barhl1a:GFP is expressed in RGCs and their post-mitotic precursors (Fig. 2E-G').

To further investigate expression of $\mathrm{Tg}($ barhl1a:GFP) with respect to atoh7 we generated a $\mathrm{Tg}($ barhl1a:GFP ;atoh7:gap43-RFP) double transgenic line in which the Atoh7:gap43-RFP labels all atoh7-expressing progenitors and their derived retinal cell types, including barhl2-expressing amacrine cells ${ }^{20,28}$. Studies have shown that Atoh7:gap43-RFP becomes first visible in the anterior ventral retina already at 27-28 hpf, underlying the onset of retinal neurogenesis ${ }^{20,28,29}$. Consistently, GFP expression from the Barhlla reporter could be seen appearing at $\sim 30 \mathrm{hpf}$, hence with two or three hours delay after the onset of atoh7:gap43-RFP expression (Fig. 3A-A"). Likewise, consistently with the idea of Barhl1a:GFP being restricted to post-mitotic RGCs, GFP expression was always seen in the basal half of the neuroepithelium whilst it remains excluded from the retinal apical surface where mitosis takes place (Fig. 3B-D" and 4A-A'). At $50 \mathrm{hpf}$, the GFP signal remains strong in the RGCs and their axons that have extended along the optic tract (Fig. 4C,D" and Supplementary Movie 2).

Imaging in the retina of $\mathrm{Tg}$ (barhl1a:GFP;atoh7:gap43-RFP) embryos also revealed that not all Atoh7:gap43-RFP cells in the GCL appear as Barhlla:GFP positive, as it would be expected if Barhlla labels a subpopulation of RGCs (see asterisks in Figs. 2G', 4A-A',C-C"). To assess this, Barhlla:GFP positive cells were counted in the central retina of $50 \mathrm{hpf}$, Tg(barhl1a:GFP; atoh7:gap43-RFP) embryos immunostained against GFP and Zn5 (also known as Alcama/DM-GRASP/Neurolin/Zn $8^{30}$ ), a cell adhesion molecule that is transiently found on the entire surface of differentiated RGCs, and is therefore a suitable marker for labeling all RGCs ${ }^{30}$. Quantification of the Barhl1a:GFP nuclei shows that, on average, only about 54\% of the RGC population (1391 cells, $\mathrm{n}=7$ retinae) is also Barhlla:GFP positive (Fig. 4B and Supplementary Table 1).

Besides RGCs, the GCL also contains displaced amacrine cells. These displaced amacrine cells are however present in very low numbers, which would not largely affect the percentage of Barhl1a:GFP RGCs. We aimed nevertheless to rule out the possibility that some of the GFP-negative cells in the GCL may be displaced amacrine cells. To this end we generated a Tg(barhl1a:GFP;ptf1a:dsRed) double transgenic line, in which the ptf1a:dsRed transgene labels all amacrine and horizontal cells ${ }^{31-33}$ (Fig. 5). Analysis of the retinae of Tg(barhl1a:GFP;ptf1a:dsRed) embryos at $50 \mathrm{hpf}$ revealed that expression of the two reporter genes are mutually exclusive, with all displaced amacrine cells expressing the Ptfla reporter being Barhl1a:GFP-negative (Fig. 5). This further confirms that expression of barhlla is restricted to a RGC population.

We conclude that barhlla turns on in about half the RGC precursors. The other half of the atoh7-expressing RGC precursors would then differentiate without the direct contribution of Barhlla in the retina. 


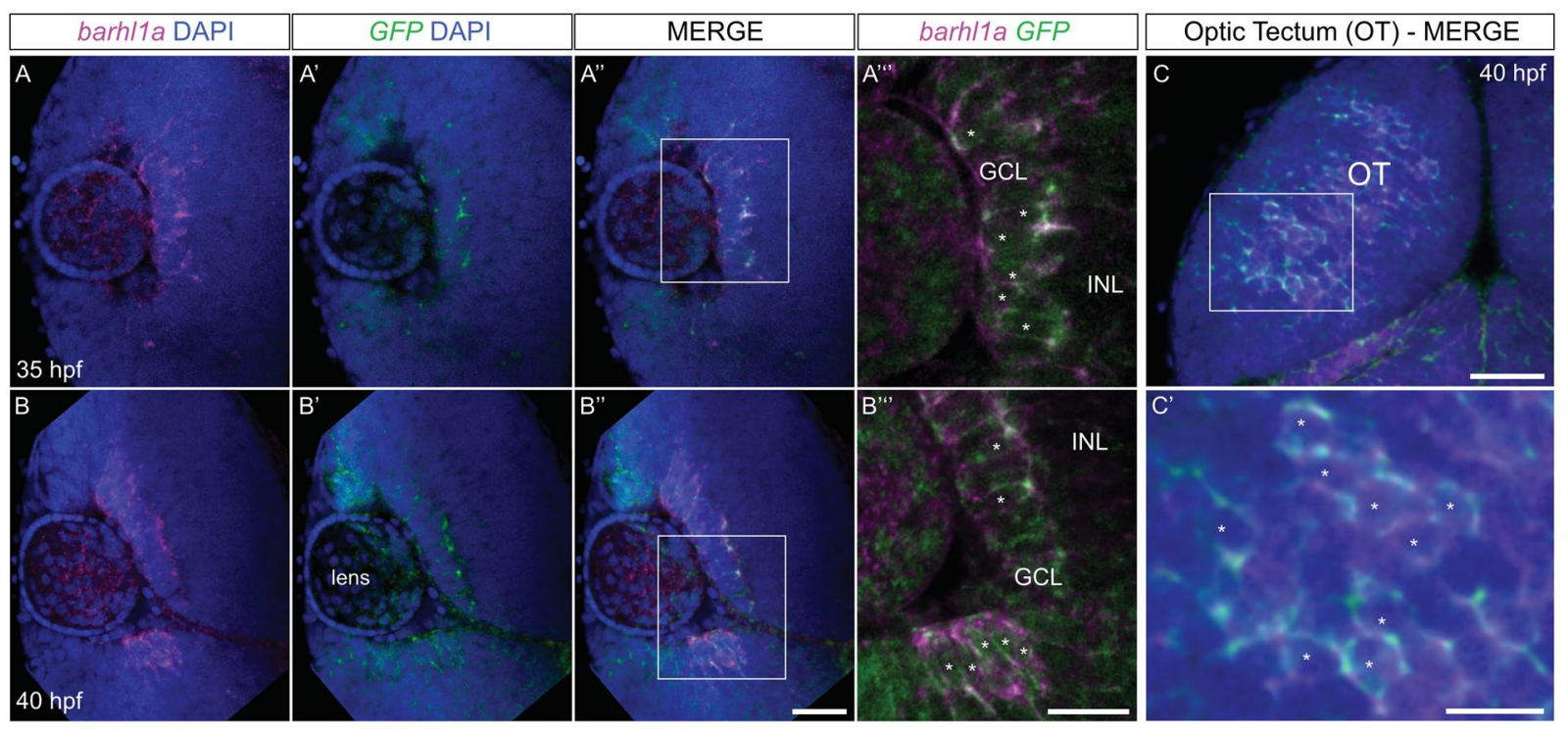

Figure 1. Expression of endogenous barhlla is reflected by barhl1a:GFP in the retina and brain. (A-B'") Confocal optical section in frontal view (dorsal is to the top) through the retina of a $35 \mathrm{hpf}\left(\mathrm{A}-\mathrm{A}^{\prime \prime}\right)$ and $40 \mathrm{hpf}$ (B - B'") embryo after FISH against endogenous barhl1a (A, B in magenta) and the GFP transgene (A', B' in green). Retinae are counterstained with DAPI (blue). (A", B") merging of the two channels shows co-localization of the two transcripts in the GCL. (A"', B"') magnifications of (A") and (B") (white squares), respectively, without the DAPI channel. (C-C') Confocal imaging into the tectum of a $40 \mathrm{hpf}$ embryo after FISH and DAPI conterstaining shows co-localization of barhlla with GFP. Images represent a Z-stack in dorsal view (anterior is to the top) into the optic tectum (OT). (C') magnification of (C) (white square). Asterisks highlight regions with colocalizing signals. GCL:

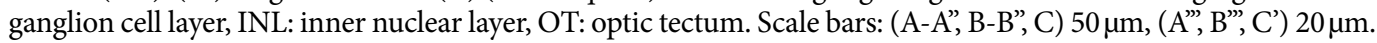

Expression of the Barhl1a reporter in the brain labels diencephalic commissural neurons and their mitotic precursors. Prior to Barhlla reporter activity in the retina, Barhlla:GFP-positive cells can be seen starting from 10-14 somites in the forebrain, in locations corresponding to the previously reported expression of barhl1 mRNA in diencephalic domains of mammals, Xenopus and fish ${ }^{1,3,4,10,34}$ (Fig. 6A,B-B' and Supplementary Movie 3). These diencephalic domains have been shown to encompass the prospective preoptic area, thalamus and posterior tuberculum/posterior hypothalamus ${ }^{1,3,4}$. Subsequent GFP signals extend to more posterior domains, such as the ventral midbrain, pretectal/tectal area and the hindbrain ${ }^{3,4,35}$ (Fig. 6C-C" and Supplementary Movie 4). Finally, imaging of the forebrain of $\mathrm{Tg}$ (barhl1a:GFP) embryos additionally revealed previously not reported barhl1a expression in few cells likely corresponding to pituitary cells (Fig. 6B-B' and Supplementary Movie S4).

The Barhlla reporter line offers the advantage to visualize not only the development of neuron cell bodies in the retina and brain but also of their axonal processes in vivo (Fig. 6). Imaging analysis of the brain of $\mathrm{Tg}$ (barhl1a:GFP) embryos indeed revealed, besides the optic chiasm, other two early commissural tracts deriving from barhlla-expressing cells. A first bundle of GFP positive fibers become visible at around 20-22 hpf, extending on both sides of the ventral-anterior diencephalon towards the midline, and forming a commissure around the presumptive optic tract (Fig. 6B-B' and Supplementary Movie 5). The first RGCs become postmitotic in the retina only at around $27 \mathrm{hpf}^{26}$; we can therefore exclude that these Barhlla:GFP-labelled axons crossing the ventral midline are RGC axons. Time-lapse imaging analysis using a lateral view further highlighted the temporal and spatial dynamics of the development of this ventral commissure in $T g$ (barhl1a:GFP) embryos, which appear before the optic chiasm and is located immediately posterior and dorsal to it (Supplementary Movie 6 and see also Supplementary Movie 4). These observations are consistent with Barhl1a:GFP-positive ventral commissure likely contributing to the previously described post optic commissure (POC) $)^{36-39}$. We then generated a BAC-based barhl1a:gal4 (barhl1.2:gal4-vp16) DNA construct and injected it in one-cell stage Tg(UAS:RFP;cry:GFP) embryos, to obtain mosaic expression of RFP (Supplementary Movie 7). Single Barhlla:gal4;UAS:RFP cells were seen extending their fibers along this POC; which are likely located in the caudal diencephalon, where commissural neurons have been previously described (Supplementary Movie 7) ${ }^{36-42}$. Furthermore, we found that barhlla expression in this region overlaps with the expression of $\operatorname{lm} x 1 b 1$, a marker of the presumptive caudal diencephalon/posterior tuberculum in which commissure neurons have been reported (Supplementary Fig. S1B-B") ${ }^{43,44}$. Remarkably, once the POC is fully formed and the optic nerves have reached their final destination, the Barhl1a:GFP commissural fibers are no longer visible (Fig. 4D-D" and Supplementary Movies 2). Therefore, the developmental spatial-temporal time of the appearance of the barhl1a:GFP expression in the POC tightly correlates with the formation of the optic tract.

To further investigate the location of the Barhl1a:GFP-labeled diencephalic commissure with respect to the optic chiasm, we performed imaging in the brain of $\mathrm{Tg}$ (barhl1a:GFP;atoh7:gap43-RFP) embryos at 35 hpf (Fig. 6C-E' and Supplementary Movie S4). At this developmental stage, Atoh7:gap43-RFP -positive RGC 


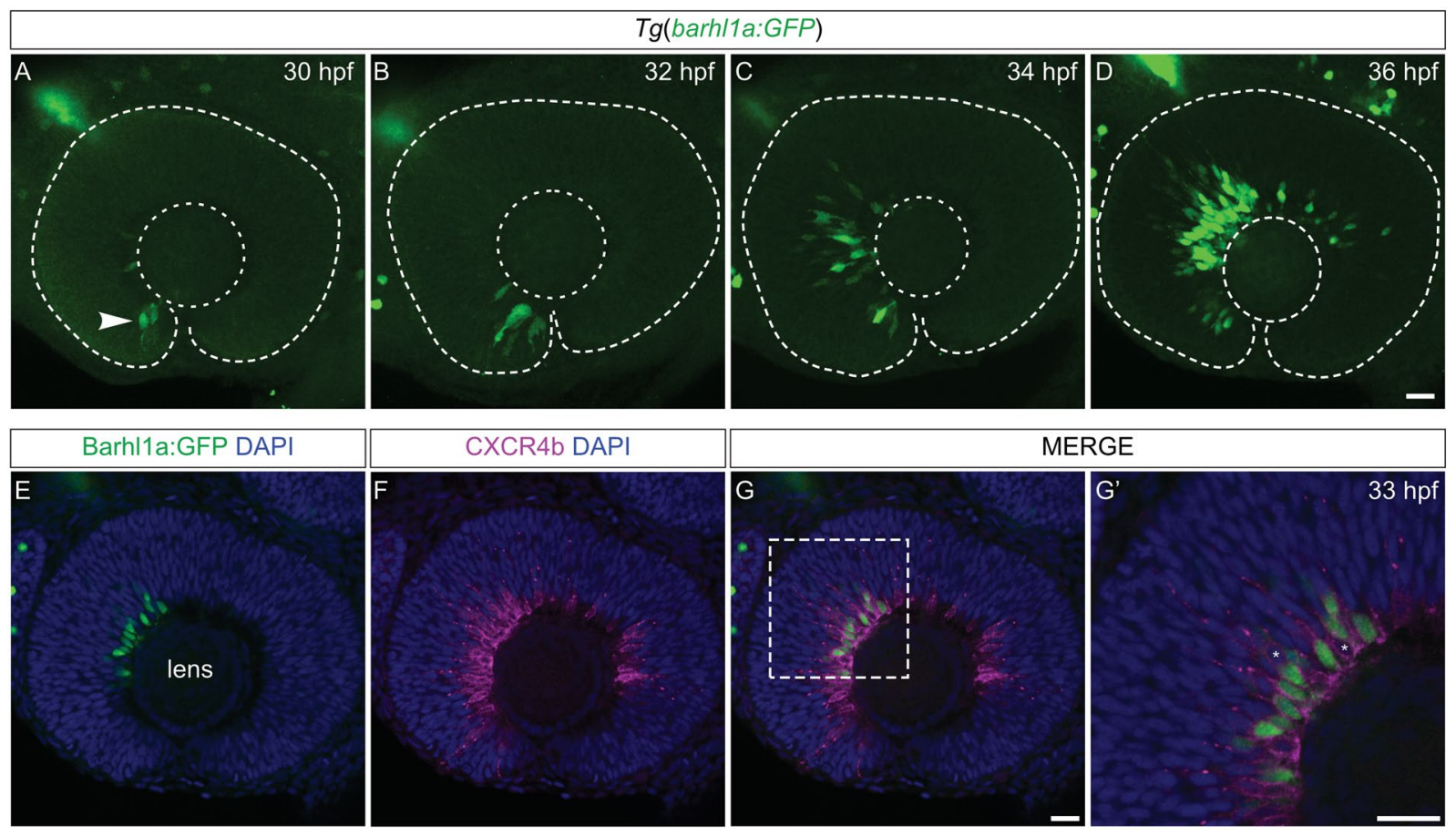

Figure 2. $\operatorname{Tg}($ barhl1a:GFP) labels new-born RGCs. (A-D) Spatial-temporal distribution of $\operatorname{Tg}($ barhl1a:GFP) expression in three different retinal developmental stages at 30 (A), 32 (B), 34 (C) and 36 (D) hpf. The GFP signal (green) becomes first evident at $\sim 30 \mathrm{hpf}$ in the ventral retina (white arrow in A) and subsequently follows the spatio-temporal wave of RGC differentiation. All images represent $1 \mu \mathrm{m}$ Z-stacks in lateral view. (E-G') Single $1 \mu \mathrm{m}$ optical slice (Z-stack) of a $33 \mathrm{hpf} \mathrm{Tg}$ (barhl1a:GFP) embryo immunolabeled with anti-Cxcr4b (magenta) and anti-GFP (green) antibodies. (E) GFP is detected in cells located in the basal half of the neuroepithelium around the lens at $33 \mathrm{hpf}$. These are also labelled by Cxcr4b (magenta in F, G and zoomed insert G'), which mark postmitotic RGCs and their precursors at the cell membrane. Asterisks in G' highlight Cxcr4b-positive (GFP-negative) RGCs. All images represent lateral view, anterior is left, dorsal is top. Scale bars: $20 \mu \mathrm{m}$.

axons can be clearly identified; which exited the retina and crossed the ventral midline to form the optic chi$\operatorname{asm}^{45}$ (Fig. 6C'D”). Conversely, only very few of these gap43-RFP -positive axons are also Barhl1a:GFP-positive (Fig. 6C-C"), further suggesting that the birth and/or maturation of Barhlla:GFP RGCs is delayed with respect to the first wave of RGC genesis. Rotating the reconstructed 3D volume further highlighted the two contiguous but distinct commissures identified by the Atoh7 and Barhl1a reporters, respectively (Fig. 6D-D"). Lastly, Tg(barhl1 a:GFP; atoh7:gap43-RFP) embryos were co-labeled immuno-histochemically with antibodies against acetylated Tubulin and GFP. The GFP signal overlaps, albeit only partially with that of acetylated Tubulin in the POC fibers (Fig. 7), further indicating that the Barhl1a:GFP cell projections contribute to this diencephalic commissure.

Notably, another bundle of Barhlla:GFP positive axons could be seen at around $27 \mathrm{hpf}$, which extend dorsally and cross the midline at the presumptive rostral border of the developing tectum (Fig. 6B,C" and Supplementary Movie 6). Based on their temporal appearance and anatomical position, these commissural fibers are likely to grow along the path pioneered by the posterior commissure (PC) and might be therefore residing in the ventral midbrain or pretectal nuclei (Supplementary Movie 7) ${ }^{36,38,42}$.

In the pseudostratified neuroepithelium of the neural tube, post-mitotic neurons and glia are generated from progenitor cells that span the entire thickness of the neuroepithelium, from the basal to the apical surface. The nuclei of these progenitor cells undergo interkinetic nuclear migration, with mitotic nuclei being located at the apical surface whilst differentiating cells migrate to more basal locations ${ }^{46,47}$. Since Barhlla:GFP-positive cells span the entire thickness of the diencephalic neuroepithelium, we asked whether these cells would be found at different developmental phases of neurogenesis. $\mathrm{Tg}($ barhl1a:GFP) neurons were firstly labelled immune-histochemically with a HuC/Elav antibody labelling the cell body of differentiated neurons ${ }^{48}$. Overlap of HuC/Elav and GFP occurs in cells located further away from the apical (ventricular) surface, indicating that they are post-mitotic cells (Fig. 8A,A'). Conversely, GFP positive cells immediately adjacent to the apical ventricular surface expressed the mitotic marker phospho-histone $3(\mathrm{pH} 3)^{49}$, indicating that they are mitotic precursors, as well as could be seen undergoing mitotic divisions (Fig. 8B-B'). Neuronal progenitors in the zebrafish neuroepithelium have been reported to express markers of radial glia, such as the glial acidic fibrillary protein (GFAP) ${ }^{48,50}$. Mosaic expression of an $g f a p: l s s-k a t e$ construct, where the promoter of $g f a p$ has been cloned upstream of the lss-mKate1 gene, highlighted GFAP:Lss-mKate1/Barhlla:GFP double positive cells at different apical-basal distances within the thickness of the neuroepithelium (Fig. 8C). 
$\mathrm{t}=0^{\prime}(30 \mathrm{hpf})$
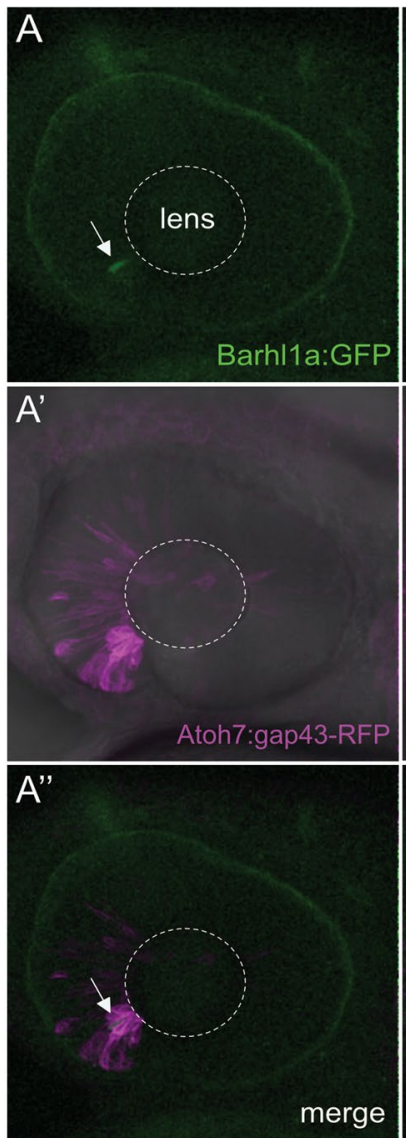

$\mathrm{t}=300^{\prime}(35 \mathrm{hpf})$
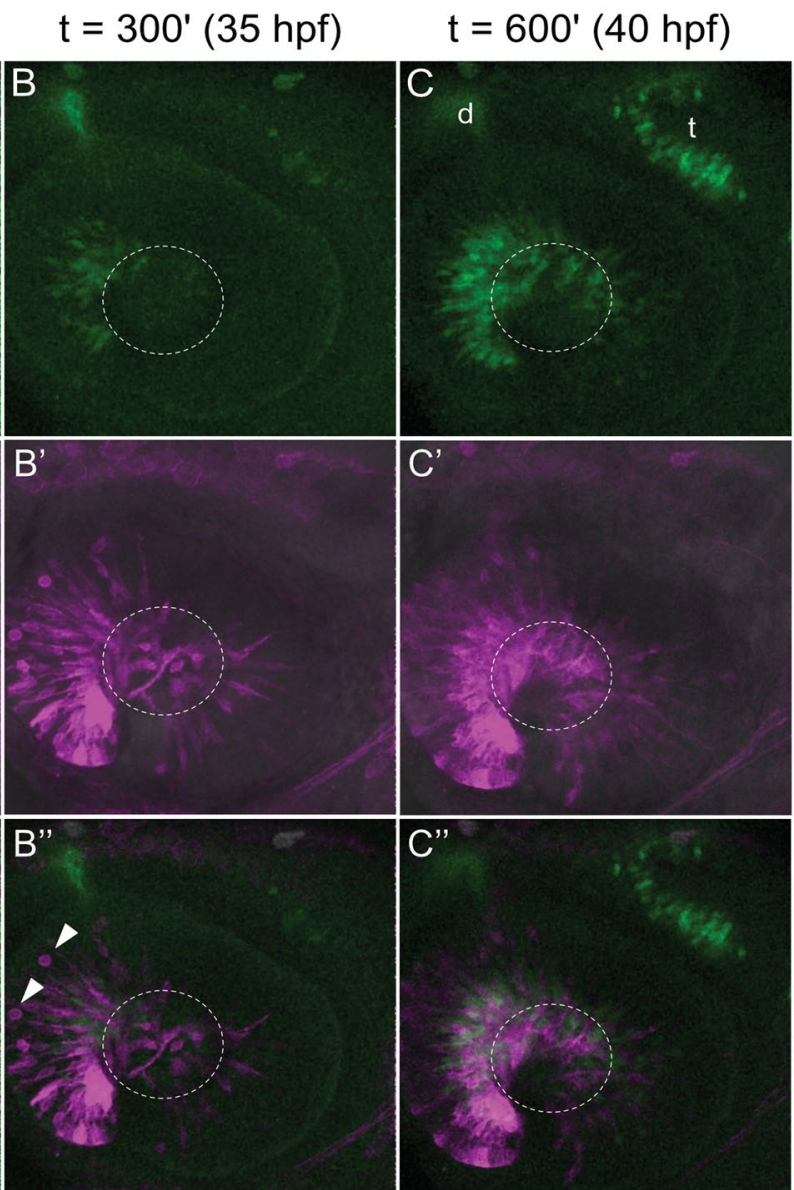
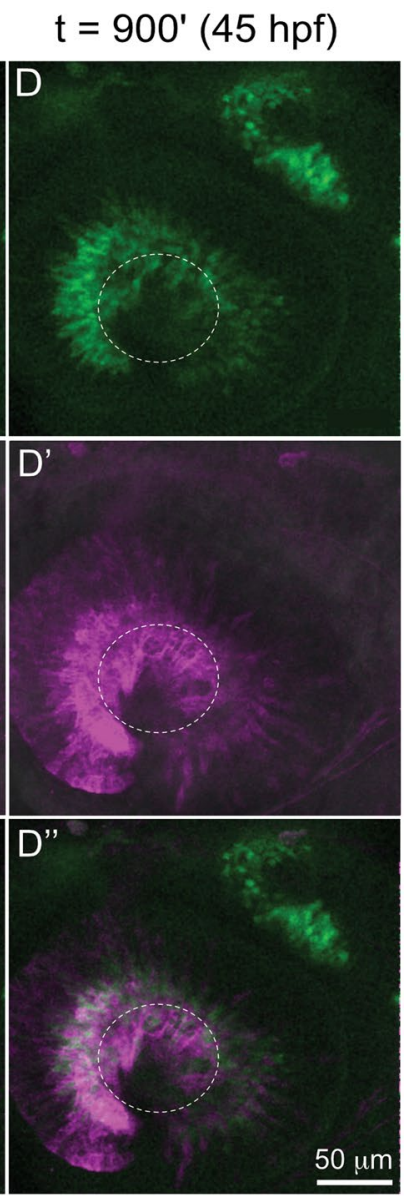

Figure 3. Time course of barhl1a:GFP;atoh7:gap43-RFP expression. Frames from a time-lapse movie in the retina of a Tg(barhl1a:GFP; atoh7:gap43-RFP) double transgenic embryo imaged from 30 hpf to $45 \mathrm{hpf}$. Each timeframe represents a projection of Z-stacks in lateral view. Anterior is to the left, dorsal is to the top. Time-points $(\mathrm{t})$ are $\mathrm{t}=0$ minute (') at $30 \mathrm{hpf}\left(\mathbf{A}-\mathbf{A}^{\prime \prime}\right), \mathrm{t}=300^{\prime} \sim 35 \mathrm{hpf}\left(\mathbf{B}-\mathbf{B}^{\prime \prime}\right), \mathrm{t}=600^{\prime} \sim 40 \mathrm{hpf}\left(\mathbf{C}-\mathbf{C}^{\prime \prime}\right)$ and $\mathrm{t}=900$ ' $45 \mathrm{hpf}\left(\mathbf{D}-\mathbf{D}^{\prime \prime}\right)$. The first GFP-positive cells (in green) can be detected at $\sim 30 \mathrm{hpf}(\mathrm{t}=0)$ in the anterior-ventral retina (white arrow in $\left.\mathrm{A}, \mathrm{A}^{\prime \prime}\right)$, when the Atoh7:gap43-RFP signal (in magenta) has already spread across the nasal retina at this developmental stage. The wave of Barhlla:GFP follows the wave of Atoh7:gap43-RFP across the dorsal and temporal retina (B-D"), always remaining confined to the basal half of the retinal neuroepithelium. White arrowheads in (B") point at RFP-positive (GFP-negative) cells rounding up at the apical surface before mitotic division. Barhlla:GFP expression can also be observed in the developing optic tectum $(\mathrm{t}, \mathrm{C})$ and diencephalon $(\mathrm{d}, \mathrm{C})$. The dotted circles highlight the position of the lens. Scale bar: $50 \mu \mathrm{m}$.

We conclude that, unlike in the neural retina, where barhl1a expression is turned on post-mitotically in RGC precursors, expression of the Barhlla reporter in the diencephalon marks populations of progenitor/precursor cells undergoing cell division and neuronal and glial differentiation.

In summary, the observed spatial-temporal dynamics of Barhl1a:GFP are consistent with Barhl1a being a subtype identity factor for populations of commissural neurons in the brain as well as in the retina. The Barhlla reporter indeed specifically labels particular neuronal subsets in the forebrain and their extending axons, which form commissures across brain hemispheres. These barhl1a:GFP-expressing axons follow pre-existing embryonic commissures: the POC, the PC and the optic chiasm in a stereotypic pattern that is reminiscent of the previously described developmental sequence of the formation of archetypal tracts $36,37,51,52$.

\section{Discussion}

Barhl genes have a crucial role in the control of neuronal subtype acquisition and maintenance during development and in the adult ${ }^{1,2,5,6,8,15,53}$. Loss of Barhl2 protein in the postnatal mammalian retina causes programmed cell death of more than $50 \%$ of $\mathrm{RGCs}^{14}$. Therefore, a better understanding of the Barhl function is essential to prevent RGC death and/or enable RGCs and axon regeneration. However, the mammalian Barhl2 is concomitantly expressed in amacrine cells and it is required for their subtype identity acquisition ${ }^{14,15}$. Untangling these two concurrent functions of Barhl2 might therefore be challenging in mammals. Barhl proteins play evolutionarily conserved roles in retinal cell type maturation ${ }^{13,20}$. Furthermore, the two zebrafish barhl paralogues genes, 

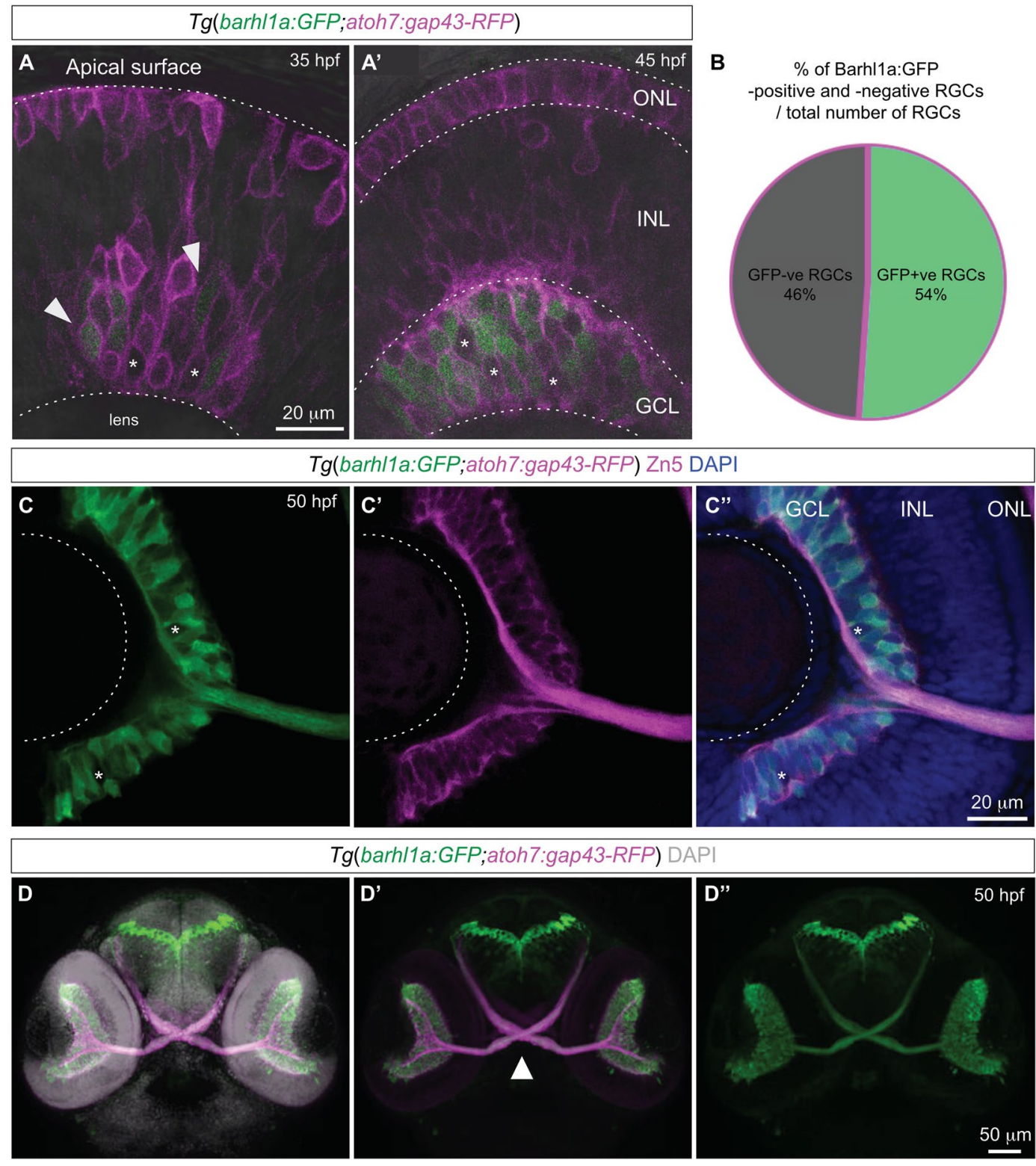

Figure 4. Barhlla:GFP labels a population of new-born RGCs and their axons. (A, A') Single optical slices (Z-stacks) extracted from a movie frame of a retina of a $\operatorname{Tg}$ (barhl1a:GFP;atoh7:gap43-RFP) embryo at 35 and $\sim 45 \mathrm{hpf}$, respectively, showing the distribution of Barhlla:GFP (green) and Atoh7:gap43-RFP (magenta) cells across the basal-apical surface of the retinal neuroepithelium. For both images anterior is to the left, dorsal is to the top. The outline of the retina is shown in the gray channel. Asterisks highlight the GFP-negative (gap43-RFP -positive) cells whereas the arrowheads point at the GFP positive cells. (B) Quantification of the percentage of GFP-positive (GFP + ve) and GFP-negative (GFP-ve) cells performed at $50 \mathrm{hpf}$ revealed that $54 \%$ of RGCs are expressing Barhlla:GFP (1391 total counted cells from $n=7$ retinae). (C-C”) A single optical slice (Z-stack) in frontal view (dorsal is top) through the retina of a $50 \mathrm{hpf}, \mathrm{Tg}$ (barhl1a:GFP; atoh7:gap43-RFP) embryo counterstained with DAPI (C”), anti-GFP (C, green) and the RGC marker anti-Zn5 (C', magenta) to brightly label both Barhlla:GFP and the GCL. Asterisks highlight Barhl1a:GFP-negative (gap43-RFP/Zn5 -positive) RGCs. (D-D") Frontal view of a 3D reconstruction of the head of an Tg(barhl1a:GFP;atoh7:gap43-RFP) embryo at 50 hpf (dorsal is to the top). Atoh7:gap43-RFP labelled retina and optic nerve are shown in magenta. At this stage, Barhlla-GFP cells (green) have fully differentiated and extended their axons out of the retina forming the optic nerve. The optic nerves cross contra-laterally to form the optic chiasm (white arrowhead, D') and reach their targets in the brain. The embryo has been counterstained with DAPI as shown in gray in (D). ONL, outer nuclear layer; INL, inner nuclear layer; GCL, ganglion cell layer. Scale bars: (A-A') $20 \mu \mathrm{m}$, (C-C”) $20 \mu \mathrm{m}$, (D-D”) $50 \mu \mathrm{m}$.

barhll $a$ and barhl2, exhibit complementary expression in the retina ${ }^{10}$, which together resemble mammalian Barhl2 expression.

We here started to explore whether it could be possible to disentangle the dual function of the single mammalian Barhl2 gene in the retina, by analyzing its zebrafish orthologous genes. With the generation of the 
Tg(barh/1a:GFP;ptf1a:dsRed)
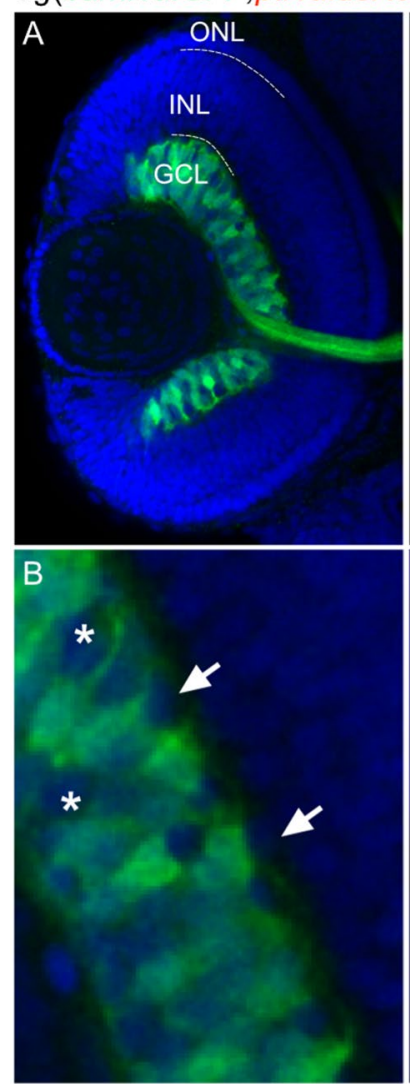
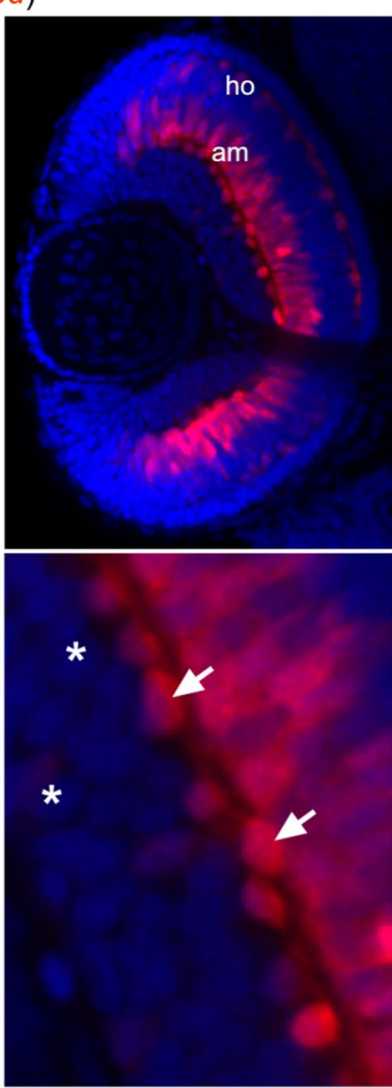
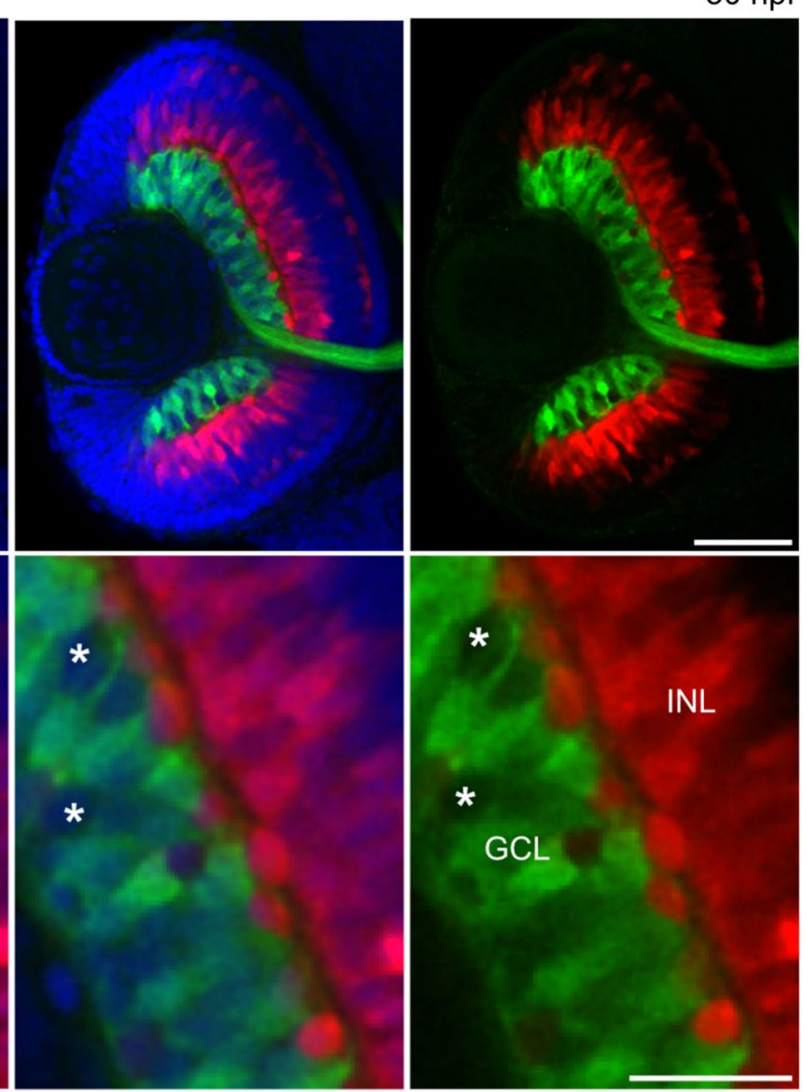

Figure 5. Barhlla and Ptfla reporter expressions are mutually exclusive. (A-B) Confocal imaging through the retina of a $50 \mathrm{hpf} T g$ (barhl1a:GFP;ptf1a:dsRed) embryo fixed and counterstained with DAPI (blue). Each image represents a single Z-stack in frontal view. Anterior is to the top. (B) always represents a higher magnification of (A). GFP-positive (green) cells are detected in the GCL whilst DsRed-positive cells (red) are detected in the amacrine (am) cells and horizontal (ho) cells of the inner nuclear layer (INL). White arrows point at DsRedpositive amacrine cells displaced in the GCL; which are always GFP-negative. Asterisks highlight the GFPnegative cells in the GCL; which are also DsRed-negative (B). GCL, ganglion cell layer; INL, inner nuclear layer; ONL, outer nuclear layer; am, amacrine cells; ho, horizontal cells. Scale bars: (A) $50 \mu \mathrm{m}$, (B) $20 \mu \mathrm{m}$.

$\mathrm{Tg}$ (barhl1a:GFP) transgenic line we established an essential resource. This line allowed us to provide for the first time a comprehensive description of the dynamic barhlla expression in vivo. Our study confirms and extends previous findings by showing that barhlla:GFP turns on exclusively in RGCs (most likely downstream of Atoh $7^{10}$ ). Time-lapse imaging of individual Barhlla:GFP-positive RGCs further enabled us to show that these cells are postmitotic neuro-epithelial cells, with their nuclei migrating from the apical half of the neuroepithelium towards the GCL.

Interestingly, these Barhl1a:GFP RGCs represent only about 54\% of the RGCs population, which appear to display distinct retinotectal projections. There is a delay in the appearance of Barhlla:GFP-positive axons compared to the appearance of Atoh7:gap43-RFP axons. One explanation for this delay might be that Barhlla:GFP cells comprise a population of later-born RGCs, which grow their axons along the optic tract pioneered by the early-born Atoh7-positive/Barhl2-negative RGCs ${ }^{52}$. Alternatively, the delayed onset of GFP could also reflect the requirement of barhl1a expression for the late maturation and/or maintenance of RGCs. Conclusions cannot be drawn here and future studies using the $\mathrm{Tg}($ barhl1a:GFP) line and barhl1a-inducible Gal4 constructs may be used to investigate the distinct molecular signature, morphology, dendritic patterns and retinotopic targets of Barhlla RGCs.

We have previously shown that about $58 \%$ of amacrine cells express barhl2 in the Zebrafish retina, all of which seem to arise from the asymmetric divisions of atoh7-expressing progenitors. Moreover, Zebrafish Barhl 2 is both necessary and sufficient for subtype identity acquisition of these amacrine cells downstream of Ptfla ${ }^{20}$. Given the remarkably similar temporal and subtype-restricted expression pattern of barhlla, it will be interesting in future studies to examine if and how Barhlla determines particular RGC subtype identities and retinotopic projections, and whether these subtypes tend to have recurrent clonal relationships and connectivity patterns with their lineally-related Barhl2 amacrine subtypes (Fig. 9). Lastly, the fact that Barhlla RGCs and Barhl2 amacrine cells are lineally related (that is: they arise from the same type of progenitor) suggests that these retinal cells share gene expression profiles and/or epigenetic ancestry. It will be exciting to explore whether such insights gained from a genome duplication in zebrafish will allow to narrow down the shared and distinct gene networks that are 

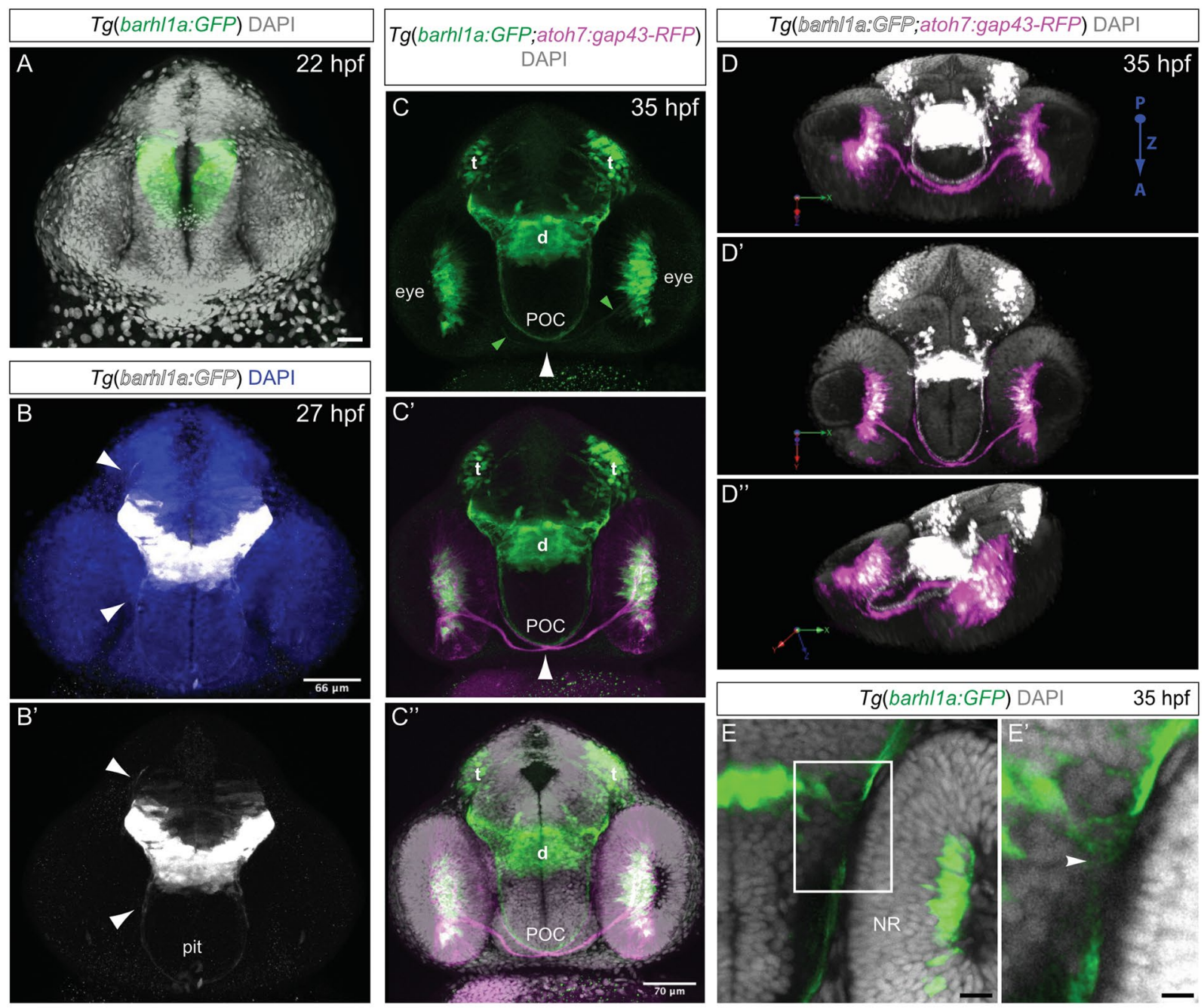

$\mathrm{F}$
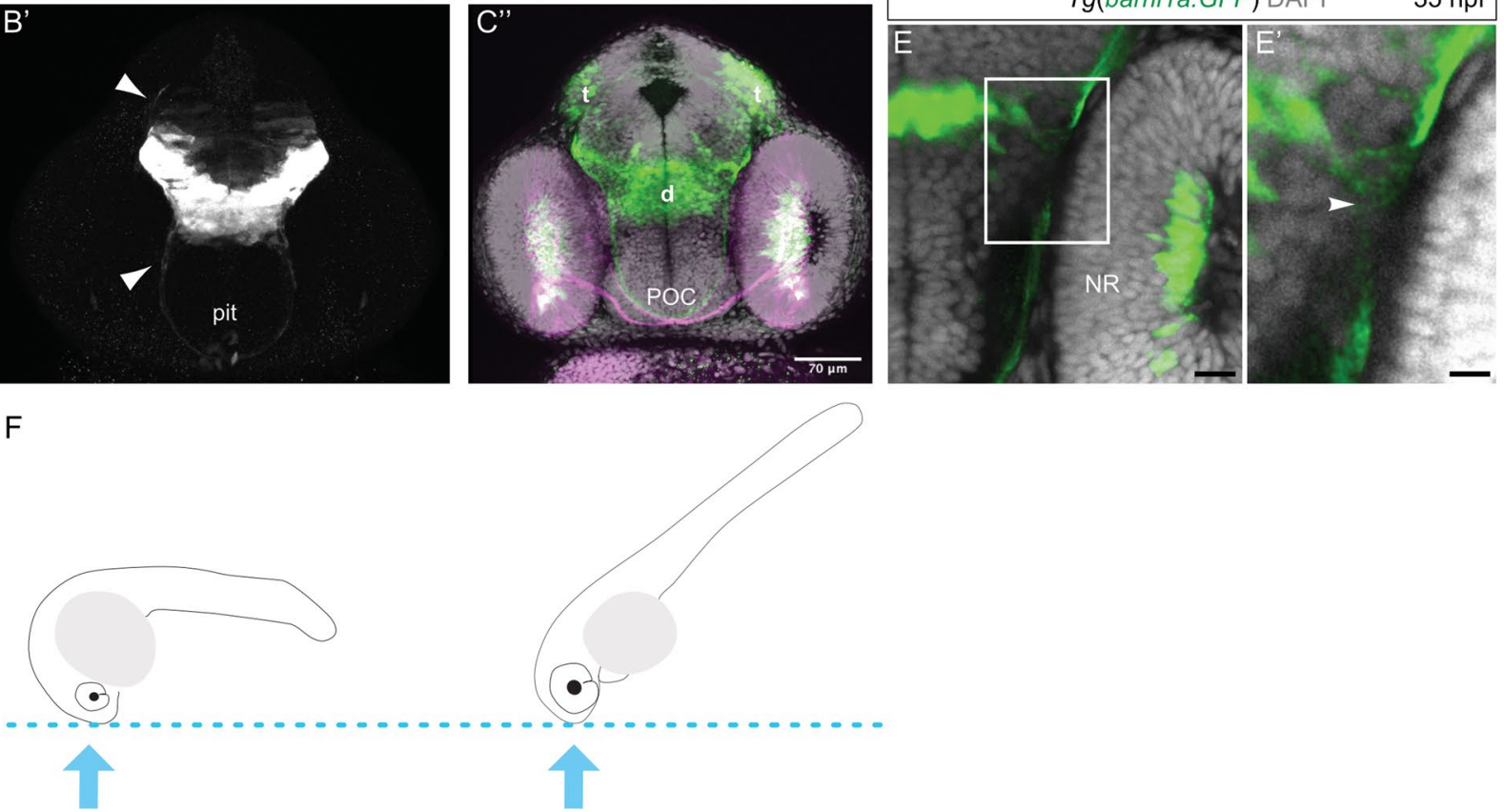

Figure 6. Developmental dynamic of Barhlla:GFP cells in the diencephalon. Images represent projections of Z-stack (A-C", E, E') and 3D reconstructions (D-D") of the anterior brain of fixed embryos at 22 (A), 27 (B-B') and $35 \mathrm{hpf}$ (C-C”, D-D" and E-E') counterstained with DAPI. Images are in frontal view, dorsal is to the top. (A) Frontal view of the anterior brain of a $\mathrm{Tg}$ (barhl1a:GFP) transgenic embryo at $22 \mathrm{hpf}$ showing Barhlla:GFP (in green) in the presumptive diencephalon. DAPI staining is shown in gray. (B-B') Frontal view of a $\operatorname{Tg}$ (barhlla:GFP) transgenic embryo at $27 \mathrm{hpf}$. DAPI in (B) is shown in blue. White arrowheads point at Barhlla:GFP projections (in gray) that extend and cross the midline ventrally and dorsally, respectively. The fibers that cross ventrally form a bundle of fibers along the presumptive optic tract. At this stage, few GFPpositive cells likely corresponding to pituitary cells can also be detected (pit, B'). (C-C”) Frontal view of an 35 hpf Tg(barhl1a:GFP; atoh7:gap43-RFP) double transgenic embryo. DAPI staining is shown in gray in C'. Barhlla:GFP cells (in green) can be seen in tectal ( $\mathrm{t}$ ) and diencephalic (d) domains as well as in the eye. Bundles of Barhlla:GFP fibers can be clearly seen crossing the ventral midline along the post optic commissure (POC). At this stage, the Atoh7:gap43-RFP positive RGCs (magenta) extend their axons out of the retina to form the optic nerve. The optic nerves cross contralaterally at the optic chiasm (white arrowhead) in close proximity to the diencephalic GFP-positive fibers forming the POC. The green arrowheads in (C) point at the few Barhlla:GFP RGC fibers (in green), which are contained within the big bundle of magenta RGC fibers shown 
in C' and C". (D-D”) 3D reconstruction and turnaround of the anterior head region of a double transgenic $\operatorname{Tg}$ (barhl1a:GFP; atoh7:gap43-RFP) embryo at $35 \mathrm{hpf}$ highlighting the optic nerves (in magenta) alongside the Barhlla:GFP fibers (in gray). DAPI staining is in gray. In all three panels the view is from the posterior side of the stack/embryo; P, posterior; A, anterior; Z, z-axis. (E-E') Enlarged confocal image (E) and its magnification (E') of a frontal view of an $T g$ (barhl1a:GFP) transgenic embryo highlighting the fibers originating from Barhlla:GFP cells located in the diencephalon (arrowheads). (F) Schematic cartoons showing approximate embryo orientation and plane of confocal imaging. Pit, pituitary. Scale bars: (A) $28 \mu \mathrm{m},\left(\mathrm{B}-\mathrm{B}^{\prime}\right) 66 \mu \mathrm{m},\left(\mathrm{C}-\mathrm{C}^{\prime \prime}\right)$ $70 \mu \mathrm{m}$, (E) $20 \mu \mathrm{m}$, (E’) $50 \mu \mathrm{m}$.

relevant for the role of Barhl in the maturation of RGCs and amacrines as well as in the survival of RGCs in the mammalian retina. Understanding this could be instrumental, for instance, for the identification of key factors whereby reprogramming of lineally-related retinal cell types, such as Barhl-dependent amacrines and RGCs, could be achieved to prevent RGC death or encourage their regeneration from amacrine cells ${ }^{54}$.

Unlike in the retina, where Barhl1a:GFP is restricted to post-mitotic neurons, barhlla:GFP in the brain is turned on in mitotic progenitors and retained in their post-mitotic daughters. Using time-lapse analysis in the brain of $T g$ (barhl1a:GFP) embryos we discovered for the first time that Barhlla:GFP in the diencephalon labels small populations of cells contributing to the POC and the PC tracts. We also found partial overlapping expressions of barhl1 $a$ and $\operatorname{lm} x 1 b 1$, a regulator of dopaminergic neurons ${ }^{44}$. It will be intriguing to assess in future studies whether these neurons co-expressing $\operatorname{lm} x 1 b 1$ and barhl1 $a$ are the ones from which such commissures arise. Also interestingly is the finding that the onset of Barhl1a:GFP-labeled POC fibers coincides with the onset of RGC axon maturation and is transient, i.e. it disappears when the optic tract is fully differentiated. Lastly, it was also intriguing to find that, whilst the Barhlla:GFP labelled POC neurons also express GFAP, a marker of progenitors and glial cells ${ }^{48,50}$, the Barhlla:GFP labelled POC fibers only partially co-localize with the POC axonal marker acetylated tubulin. Future studies will assess the precise (glial and/or neuronal) identity of Barhl1a:GFP commissural fibers; they will also assess whether the remarkably harmonized developmental pattern between the Barhl1a:GFP-labeled POC and optic chiasm may underlie a molecular cross-talk required for the proper formation of the optic tract.

In conclusion, our novel experimental tools and insights into the spatial-temporal dynamics of Barhlla:GFP in the retina and brain provide a fundamental framework for further investigation of Barhlla-specific RGCs and their retinotectal RGC projections as well as of Barhlla-commissural projection neurons in vivo. Such investigations are likely to be relevant to dissect BarHl functions in mammals and to understand and ameliorate the pathophysiology of BarHl-linked diseases.

\section{Methods}

Animals and ethic statement. Fish were maintained at $26-28^{\circ} \mathrm{C}$ and embryos raised at $28.5^{\circ} \mathrm{C}$ and staged as described previously ${ }^{55,56}$. Fish were housed in two facilities: Fish facility at COS, University of Heidelberg, Germany; fish facility at CIBIO, University of Trento, Italy. Each facility is under supervision of and in accordance with local animal welfare agencies and European Union animal welfare guidelines (Tierschutzgesetz 111, Abs. 1, Nr. 1; Regierungspräsidium Karlsruhe and the Italian Ministry of Health - permit no.: 151/2019-PR). As zebrafish sex cannot be determined until they have reached age of reproduction, embryos of either sex were used exclusively before free-feeding stages. Embryos used for whole-mount imaging were treated with $0.0045 \%$ 1-phenyl-2-thiourea (Sigma) to delay pigment formation. Lines used in this study were generated in the zebrafish wild type background $(\mathrm{AB} / \mathrm{AB}$ or $\mathrm{AB} / \mathrm{WIK})$. The fish facility is under the supervision of the local representative of the Animal Welfare Agency.

Fish lines and constructs. For the generation of the $\mathrm{Tg}$ (barhl1a:GFP) line, the BAC clone CH120-215H7 (CHORI, BACPAC, Oakland, CA, USA) was modified using the flpe recombinase system in EL250 cells ${ }^{57}$. The coding sequence of eGFP with a SV40 polyA site was inserted within the first exon of the barhl1.2 genomic sequence and the tol2-AmpR cassette was inserted in the TARBAC2.1 backbone by PCR. The modified BAC was injected together with tol 2 mRNA into one cell-stage zebrafish embryos to generate a stable transgenic line.

Along with the newly generated and validated $\mathrm{Tg}($ barhlla:GFP) line, five other transgenic lines expressing GFP, dsRed and gap43-RFP under the control of different promoters were used in this study: the previously published $\mathrm{Tg}$ (atoh7:gap43-RFP ${ }^{28}$; the derived outcrossed line $\mathrm{Tg}$ (barhl1a:GFP; atoh7:gap43-RFP); Tg(barhl1a:GFP;ptf1a:dsRed) generated by outcrossing Tg(barhl1a:GFP) and Tg(ptf1a:dsRed) line Tg((5.5ptf1a:DsRed)ia6) previously published ${ }^{20}$ and the previously published $\operatorname{Tg}(U A S: R F P ; c r y: G F P)$ transgenic line ${ }^{58}$. Embryos carrying both transgenes were screened for expression of the red and green reporters using an Olympus MVX10 macrofluorescence binocular.

The barhl1a:gal4 bacterial artificial chromosome (BAC) construct was generated as follows: a BAC spanning the barhl1a genomic locus (CH211-215C18, BACPAC Resources Center) was used to perform Tol2 transposonmediated BAC transgenesis replacing gal 4 with the barhl 1 a coding sequence. Transformation through electroporation of the $p R e d E T$ plasmid was performed as described ${ }^{59}$. For Tol2 transposon-mediated BAC transgenesis, the iTol2-amp cassette ${ }^{59}$ was amplified by PCR with the primer pair pTarBAC_HA1_iTol2_fw (5' -gcgta agcggggcacatttcattacctctttctccgcacccgacatag atCCCTGCTCGAGCCGGGCCCAAGTG-3') and pTarBAC_HA1_ iTol2_rev (5'-gcggggcatgactattggcgcgccggatcgatccttaattaagtctactaATTATGATCCTCTAGATCAGCTC- $\left.3^{\prime}\right)$, where the lower and upper cases indicate the pTarBAC2.1 sequences for homologous recombination and the iTol2-amp annealing sequences, respectively. Subsequently, the amplified iTol2-amp cassette was introduced into the backbone ( $p$ TarBAC2.1) of the Barhl1a-BAC. $500 \mathrm{ng}$ of the PCR product ( $1 \mathrm{~mL}$ ) were used for electroporation. 

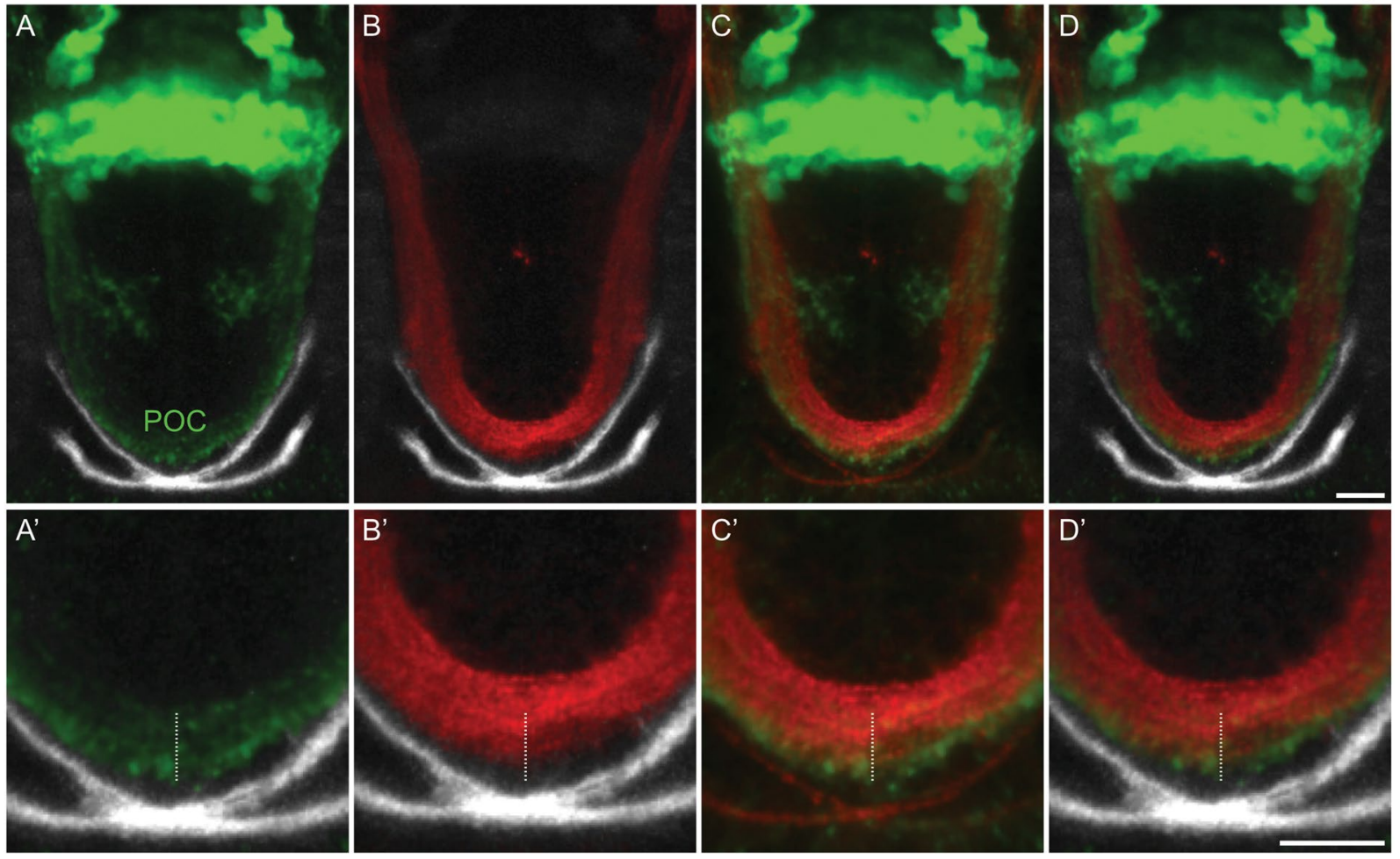

Figure 7. Barhl1a:GFP fibers co-localize with the neuronal projection marker acetylated Tubulin, a marker for the POC. (A-D) single optical sections (Z-stacks) through the anterior brain of a $35 \mathrm{hpf}$, Tg(barhl1a:GFP; atoh7:gap43-RFP) embryos immunostained with acetylated-Tubulin labelling the POC and its fibers (in red). Frontal view, dorsal is to the top. Barhlla:GFP cells and their projections are shown in green whilst the optic nerves labelled by Atoh7:gap43-RFP are shown in gray. The optic nerves from each eye cross at the optic chiasm site, ventral and anterior to the POC, to then proceed along the optic tract. (A'-D') higher magnification scan through the POC of the same embryo reveals partial overlap of the acetylated-Tubulinlabelled POC (highlighted by the white dashed line) and the Barhl1a:GFP fibers. Scale bars: (A-D) $50 \mu \mathrm{m}$, $\left(\mathrm{A}^{\prime}-\mathrm{D}^{\prime}\right) 20 \mu \mathrm{m}$.

$5^{\prime}$-caaaaccagtgtcataaaggacaaatgcacatttgatattgatttgactcGCCACCATGAAGCTACTGTCTTCTATCGAAC-3' and $5^{\prime}$-ctgtgagaaagtatagactcgatcccaaagctcgagccgtttgatacctcCCGCGTGTAGGCTGGAGCTGCTTC-3' primers were used to amplify and insert the gal4FF cassette into the $\mathrm{BAC}^{60}$. The lower and upper cases indicate the $\mathrm{CH} 211-$ 215C18 sequences for homologous recombination and the pGal4FF-FRT-Kan-FRT annealing sequences, respectively. $500 \mathrm{ng}$ of the PCR product $(1 \mathrm{~mL})$ were used for electroporation in Barhl1a-iTol2-amp-BAC -containing cells. The BAC DNA preparation was performed using the HiPure Midiprep kit (Invitrogen), with modifications for BAC DNA isolation as described by the manufacturer. Tol2 transposase mRNA was prepared by in vitro transcription from XbaI-linearized $\mathrm{pDB} 600^{61}$ using the T3 mMessage mMachine kit (Ambion). RNA was purified using the RNeasy purification kit (Qiagen), diluted to a final concentration of $100 \mathrm{ng} / \mu \mathrm{l}$ for injection. At least 20 injected fish were backcrossed to wild type. Germline transmission was observed in the offspring from two of such crossings; which displayed the distribution pattern of GFP as expected from barhl1a expression in the posterior thalamus and zona limitans intratalamica (ZLI) (Supplementary Fig. 1A). Only one of the two F1 generations was used to carry out all of the following analyses.

The zebrafish $g f a p$ promoter ${ }^{50}$ was derived from the Addgene plasmid: $p E G F P-g f a p$ (Intron $1 / 5^{\prime} /$ Exon1-zebrafish) (Addgene, \#39761) and $l s s m K a t e 2^{62}$ was derived from the Addgene plasmid: pLSSmKate2-N1 (Addgene, \#31867). In a first step the $g f a p$ promoter driving GFP was subcloned into a vector containing IsceI sites using NotI and XhoI restriction enzymes (NEB \#R3189 and \#R0146). To insert lssmKate2 SalI and NotI restriction enzymes (NEB \#R3138S and \#R3189) were used, resulting in the following plasmid: IsceI::gfap(Intron1/5'/ Econ1-zebrafish)::LssmKate2 polyA.

Immunohistochemistry. The primary antibodies used in this study and their dilutions were the following: chicken anti-GFP antibody (Life Technologies, A10262, 1:500), rabbit anti-DsRed (Clontech, 632496, 1:200), mouse anti-Tubulin (Sigma T5168, 1:100), mouse anti-HUC (Molecular probes, A-21271, 1:100) rabbit anti-pH3 (Millipore, 06-570; 1:500), mouse anti-Zn5 (ZIRC Zn-5; 1:200), monoclonal zebrafish anti-Cxcr4b (1:10063. Secondary antibodies were goat or donkey anti-mouse, anti- rabbit or anti-goat IgG conjugated to Alexa Fluor $488,546,594$ or 647 fluorophores (1:500 for whole-mount to 1:2000 dilutions for cryosections; Invitrogen). 

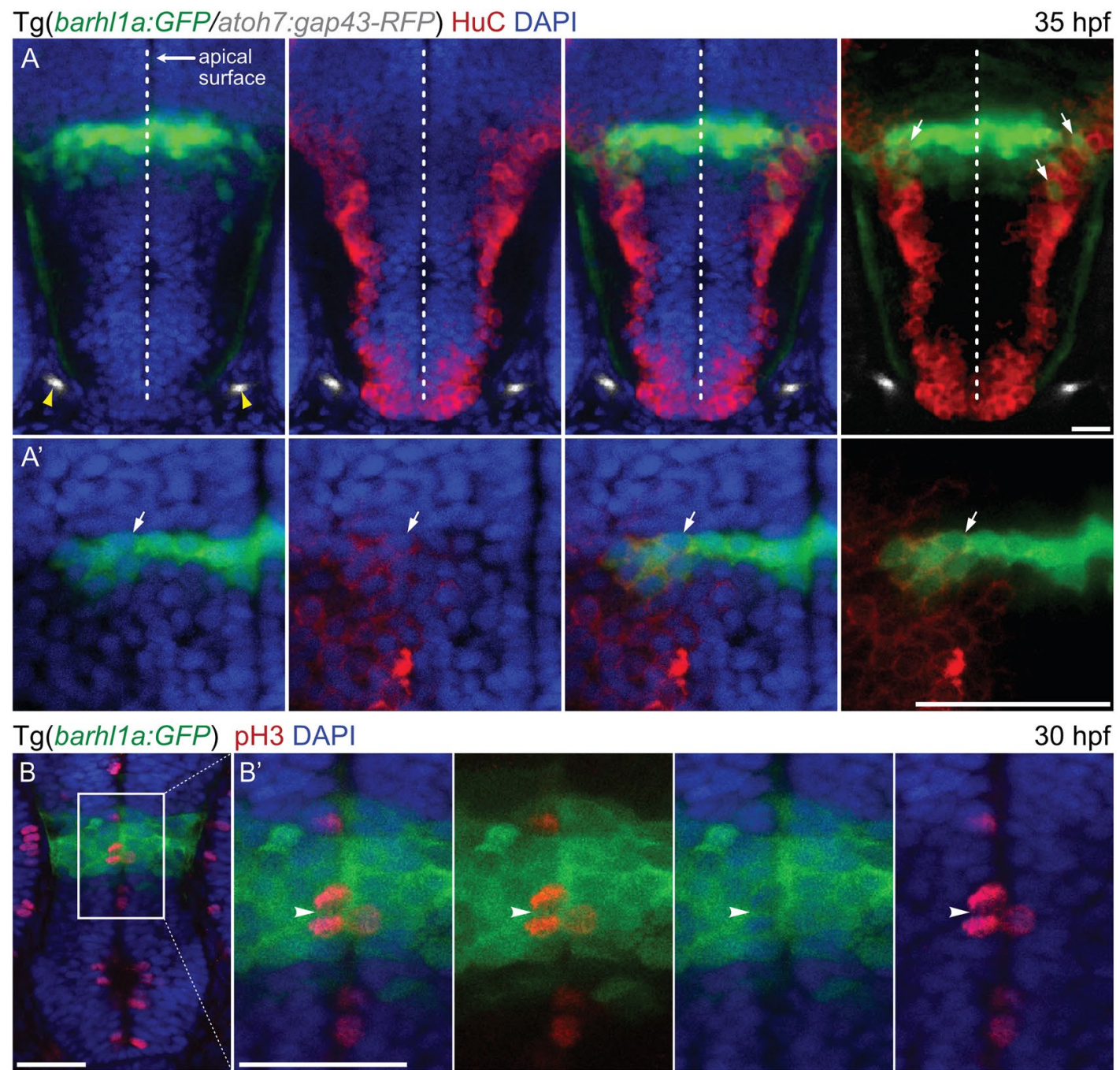

$30 \mathrm{hpf}$

Tg(barh/1a:GFP) inj.GFAP:LssKate DAPI
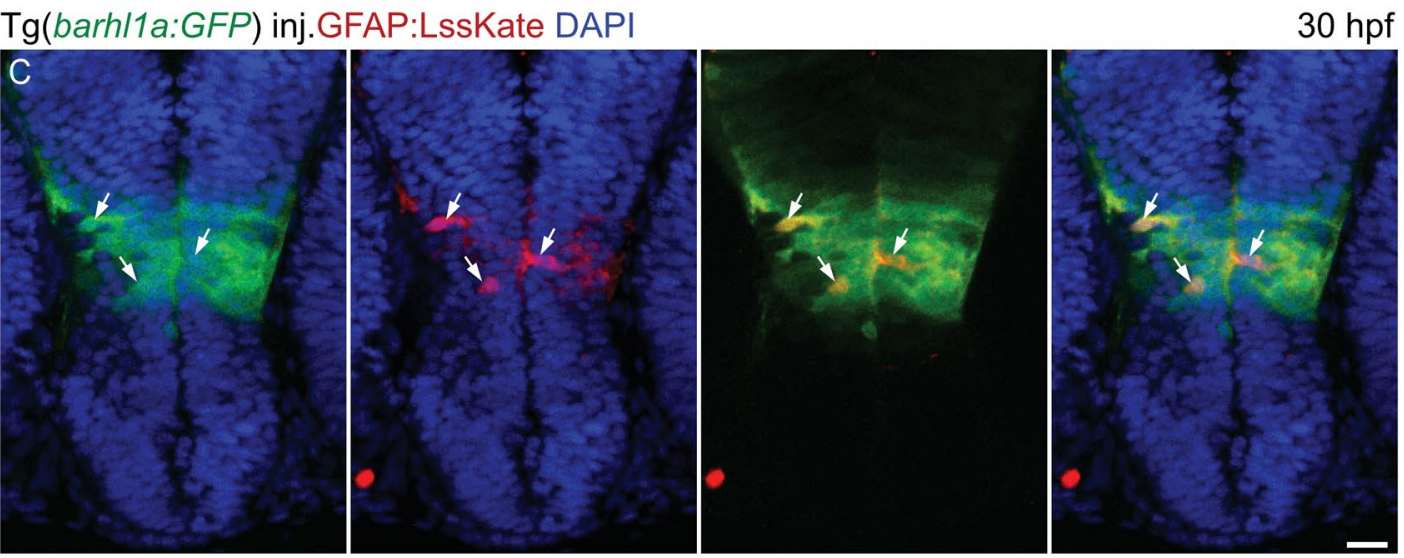

Figure 8. Barhlla:GFP in the preoptic diencephalon marks populations of mitotic progenitors and differentiating neurons. Images represent single confocal Z-stacks through the diencephalon of Tg(barhl1a:GFP;atoh7:gap43-RFP) embryos at $35 \mathrm{hpf}\left(\mathbf{A}, \mathbf{A}^{\prime}\right)$ and $30 \mathrm{hpf}(\mathbf{B}, \mathbf{C})$. Frontal view, dorsal is up. Embryos in (A-B') have been immunohistochemically stained with anti-GFP, anti-HuC (A, A') and anti-pH3 (B, B') antibodies (in red) as well as counterstained with DAPI (in blue). (A') magnifications of the images in (A) in correspondence with the barhl1a:GFP expressing cell bodies. (A) yellow arrowheads point at the optic nerves from each side marked by Atoh7:gap43-RFP (in gray). White arrows point at Barhl1a:GFP cells co-labelled with $\mathrm{HuC}$, a marker of differentiating neurons located in the basal half of the neuroepithelium. In (B) and its magnification (B'), Barhl1a:GFP cells are also co-labelled by $\mathrm{pH} 3$, which marks mitotic neurons located at the apical surface of the neuroepithelium (white arrowhead in B'). (C) $30 \mathrm{hpf} T$ g(barhl1a:GFP) embryo injected with the gfap:lssKate DNA construct shows sparse LssKate labelling in the diencephalon (in red); which colocalize with GFP (green). Scale bars: (A-C) $50 \mu \mathrm{m},\left(\mathrm{A}^{\prime}, \mathrm{B}^{\prime}\right) 20 \mu \mathrm{m}$. 
A)

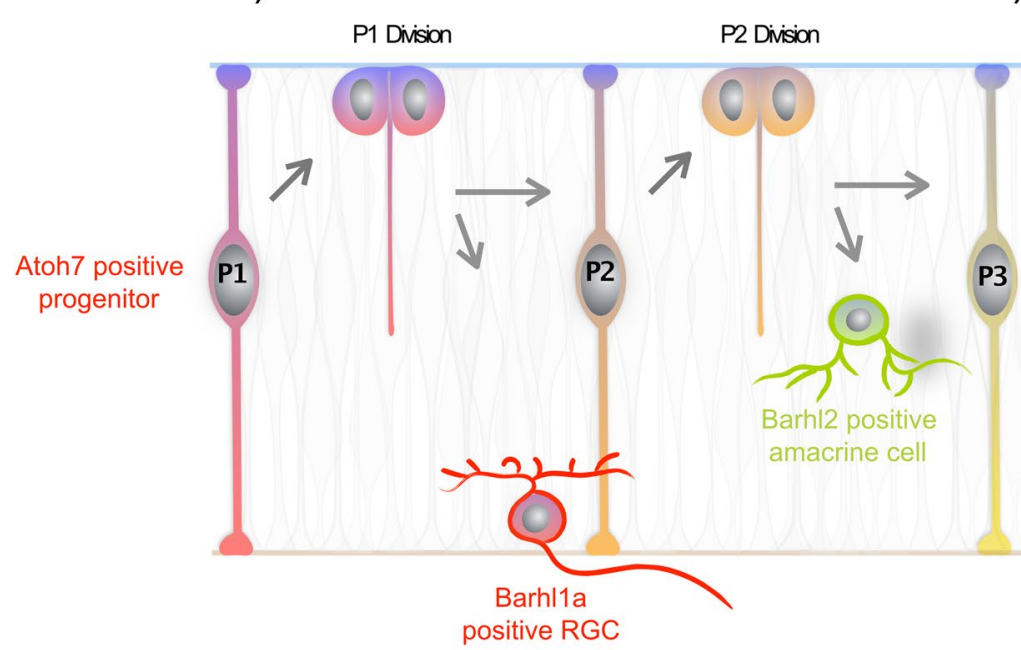

B)

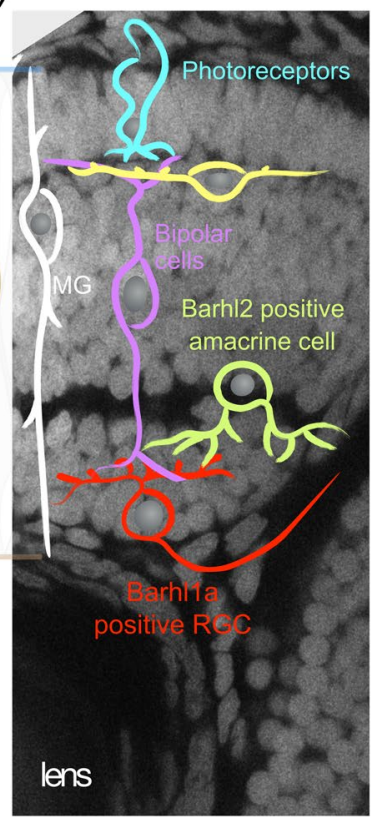

Figure 9. Hypothetical scenario describing Barhl1a RGCs and Barhl2 amacrine cells as clonally related retinal cells forming specific synapses. In this hypothetical model, (A) an asymmetric self-renewing division of an atoh7-expressing RGC progenitor gives rise to one Barhl1a-RGC and one Barhl2-amacrine cell sibling (see $\mathrm{also}^{20}$ ). (B) Cartoon representing the main retinal cell types organized into the three retinal nuclear layers; which are highlighted by the DAPI staining (in grey) of the retinal optical section from a $72 \mathrm{hpf}$ embryo in the background. MG, Müller glial cells.

Whole-mount immunohistochemistry experiments were carried out as follows: embryos were fixed 1 to 2 hours in $4 \%$ paraformaldehyde (4\% PFA). The fixative was then washed out using 1X PBS-Tween solution. Permeabilization of the embryos was done on ice using $0.25 \%$ Trypsin-EDTA (with phenol red; Gibco, Life Technologies) solution. The duration of trypsin treatment was dependent on the stage of the embryos: 28-30 hpf: $3 \mathrm{~min}$; 31-33 hpf: $4 \mathrm{~min}$; 34-35 hpf: $6 \mathrm{~min} ; 40$ hpf: 7-8 min; 45 hpf: 9-10 min; 48-50 hpf: 11-12 min; 60 hpf: 20 min. Embryos were then washed several times using $0.1 \mathrm{M}$ PBS-Tween solution (1X PBS-Tween solution), blocked using $10 \%$ blocking solution ( $10 \%$ heat-inactivated goat serum, $1 \%$ bovine serum albumin, $0.2 \%$ Triton X-100 in PBS) and incubated overnight at 4 degrees in 1\% blocking solution (10\% blocking solution in 1X PBS-Tween) in which primary antibodies were diluted. Primary antibodies were then washed out using 1X PBS-Tween and embryos were then incubated overnight at 4 degrees in 1\% blocking solution ( $1 \%$ heat-inactivated goat serum, $1 \%$ bovine serum albumin, $0.2 \%$ Triton X-100 in PBS) in which secondary antibodies and 4',6-diamidino-2-phenylindole (DAPI) were diluted. Prior to imaging, embryos were washed in $1 \mathrm{X}$ PBS-Tween and stored at 4 degrees.

For the immunohistochemistry on sections and RGC counting, 50 hpf Tg(barhl1a:GFP;atoh7:gap43-RFP) embryos were fixed in $4 \%$ paraformaldehyde (PFA) in $0.1 \mathrm{M}$ phosphate saline buffer (PBS) overnight at $4^{\circ} \mathrm{C}$, rinsed and cryoprotected in $30 \%$ sucrose (w/v) overnight at $4^{\circ} \mathrm{C}$. Embryos were then mounted vertically (head down) in freezing medium (Jung Tissue Freezing Medium, Leica Microsystems), frozen in liquid nitrogen and cryosectioned immediately with Leica CM3050 S cryostat. The thickness of sections was $14 \mu \mathrm{m}$. Sections were collected on adhesion microscope slides (SuperFrost Plus, Menzel-Gläser) and left to dry overnight at $4^{\circ} \mathrm{C}$. For immunohistochemistry, microscope slides with cryosections were washed 3 times (each wash $15 \mathrm{~min}$ ) in PTW, then covered with $10 \%$ goat blocking medium and incubated at room temperature for $1 \mathrm{~h}$. The cryosections were then incubated overnight in primary antibodies: anti-chicken GFP (Life Technologies, A10262) and anti-mouse Zn5 (Zebrafish International Resource Center (ZIRC)) both diluted 1:500 in 1\% goat blocking medium. Microscope slides were then washed again 3 times (each wash $15 \mathrm{~min}$ ) in PTW and secondary antibodies were added: anti-chicken conjugated to Alexa Fluor 488 (from donkey; Jackson ImmunoResearch Laboratories, Inc., 703-545-155) and anti-mouse conjugated to Alexa Fluor 546 (from goat; Invitrogen, A-11030) both diluted 1:500 in 1\% goat blocking medium. Cryosections were incubated with the secondary antibody mix for $1.5-2 \mathrm{~h}$ at $37^{\circ} \mathrm{C}$ in the dark and then washed again in PTW ( 3 times $15 \mathrm{~min}$ ) at room temperature. They were then incubated in DAPI $(10 \mu \mathrm{g} / \mathrm{ml}$ in PTW) for $10-15 \mathrm{~min}$ and washed in PTW (3 times $15 \mathrm{~min}$ ). Microscope slides were then dried from the back and edges with a paper tissue, $120 \mu \mathrm{L} 60 \%$ glycerol was added and cryosections were covered with coverslips $(24 \times 60 \mathrm{~mm}$, Carl Roth). Coverslips were sealed with nail polish and microscope slides were stored at $4^{\circ} \mathrm{C}$ in the dark until imaging.

Double fluorescent in situ hybridization. For barhl1a/GFP double fluorescent whole mount in situ hybridization (FISH), standard digoxigenin- and fluorescein-labeled riboprobes were combined with Tyramide 
Signal Amplification, as described by ${ }^{10}$. Barhlla riboprobe was synthesized as previously reported ${ }^{10}$ and $g f p$ riboprobe was synthesized from a linearized $p$ CS2:GFP plasmid with NotI (Fermentas or New England Biolabs) and transcribed with Sp6 (mMessage mMachine Sp6, Ambion). Incubation with the barhlla probe was for 40 min, incubation with the $g f p$ probe was for $30 \mathrm{~min}$. Embryos were then kept in the dark for all following steps. For detection and staining of the antisense probes, embryos were washed 5 times $10 \mathrm{~min}$ with TNT $(0.1 \mathrm{M}$ Tris pH7.5, $0.15 \mathrm{M} \mathrm{NaCl}, 0.1 \%$ Tween-20), incubated with $1 \% \mathrm{H}_{2} \mathrm{O}_{2}$ in TNT for $20 \mathrm{~min}$ and washed again 5 times $10 \mathrm{~min}$. Embryos were blocked in TNB (2\% DIG Block in TNT) for $1 \mathrm{~h}$ at room temperature and afterwards incubated with Anti-Digoxigenin-POD Fab fragments diluted 1:100 in TNB. For signal detection, Fluorescein (FITC), Cyanine 3 (Cy3) or Cyanine 5 (Cy5) Fluorophore Tyramide by PerkinElmer was used. Embryos were then incubated in $1 \times 4^{\prime}$,6-Diamidin-2-phenylindol (DAPI) in TNT overnight at $4^{\circ} \mathrm{C}$ and washed several times in TNT the next day. The stained embryos were then used for imaging or kept in the dark at $4^{\circ} \mathrm{C}$ until imaging.

For barhl1a/lmx1b1 double fluorescent in situ whole mount hybridization (FISH), antisense RNA probes for barhll $a$ and $l m x 1 b 1$ were produced from full-length cDNA clones generated by ${ }^{64}$. Antisense probes were generated as described in ${ }^{64}$ except that DNP-11-UTP (Perkin Elmer) was used for the production of barhl1a RNA probe. Fluorescent double whole mount FISH, RNA hybridization were performed on dechorionated $20 \mathrm{hpf}$ embryos with the tyramide amplification kit (TSA Plus Cyanine 3 System, Perkin Elmer, Boston, MA). Briefly, manually dechorionated $20 \mathrm{hpf}$ embryos were fixed in $4 \%(\mathrm{w} / \mathrm{v})$ PAF for $3 \mathrm{~h}$ at $4{ }^{\circ} \mathrm{C}$ and conserved until use in $100 \%(\mathrm{v} / \mathrm{v}) \mathrm{Methanol}$ at $-20^{\circ} \mathrm{C}$. Embryos were rehydrated through $\mathrm{MetOH} / \mathrm{PBS}$ gradient series and washed 3 times in $0.1 \%(\mathrm{v} / \mathrm{v})$ Tween 20 in PBS buffer $(\mathrm{PTW})$. Endogenous peroxidase were inactivated in $2 \%(\mathrm{v} / \mathrm{v}) \mathrm{H}_{2} \mathrm{O}_{2}$, the embryos incubated $2 \mathrm{~min}$ with proteinase $\mathrm{K}\left(10 \mu \mathrm{g} / \mathrm{ml}\right.$ at room temperature $\left(20^{\circ} \mathrm{C}\right)$, then post-fixed $15 \mathrm{~min}$ in $4 \%$ PFA for 30 minutes at room temperature and washed 5 times $5 \mathrm{~min}$ in PBS-Tween. Embryos were then pre-hybridized 3 hours before overnight incubation at $65^{\circ} \mathrm{C}$ in a hybridization buffer ( $\mathrm{pH}$ 6) containing the antisense-labeled probes. On the second day, embryos were washed in post-hybridization buffers, incubated $1 \mathrm{~h}$ in the blocking buffer and then incubated overnight with an anti-DNP-POD antibody (1:1000, Perkin Elmer). The next day embryos were washed in PBS-Tween, and stained with tyramide Cy3 solution (1:100) in $0.002 \%$ (v/v) $\mathrm{H}_{2} \mathrm{O}_{2}$ in PBS-Tween, peroxidase were inactivated in $2 \%(\mathrm{v} / \mathrm{v}) \mathrm{H}_{2} \mathrm{O}_{2}$ for 1 hour and washed in PBS-Tween. The same day, embryos were incubated overnight with an anti-digoxigenin-poly-POD antibody (1:1000, Roche), then washed in PTW and stained with tyramide Cy5 solution (1:100) in $0.002 \%$ (v/v) $\mathrm{H}_{2} \mathrm{O}_{2}$ in PBS-Tween.

Of note, FISH for GFP mRNA probe can give unspecific signals detected in the peripheral retina and lens ${ }^{20}$.

Imaging and quantification. For either imaging of fixed embryos or live imaging, embryos were embedded in $1 \%$ low-melting agarose (w/v, diluted in $\mathrm{H}_{2} \mathrm{O}$; Biozym) in $35 \mathrm{~mm}$ Glass-bottom Microwell dishes (P35G1.5-10-C, MatTek). They were oriented with truncated Microloader tips (Eppendorf) frontally (head touching the dish and body axis tilted about $45^{\circ} \mathrm{C}$ ) or laterally. After solidification, agarose was covered with a drop of water to avoid dehydration during imaging. Confocal microscopy was carried out with Leica SpE or Leica Sp5 confocal laser scanning microscope using a Leica $20 \times$ or $60 \times 1.2 \mathrm{NA}$ water-immersion objective and Leica Application Suite (LAS) software. In most cases, confocal images were taken sequentially from the whole head/retina, with a distance of 1 to $5 \mu \mathrm{m}$ in $\mathrm{z}$-direction. Optical stack of $40-60 \mu \mathrm{m}$ for time-lapse and a maximum of $100 \mu \mathrm{m}$ for fixed embryos were imaged. Live imaging was performed as previously described ${ }^{21-23}$. Images were taken every 5 or 10 minutes for $24-42 \mathrm{~h}$ and the motorized XY stage was used to image multiple embryos. For all imaging experiments, laser power was minimized as much as possible to avoid bleaching and photo-toxicity. Sequential image acquisition was also performed using individual descanned Leica PMT detectors. All image processing and 3D reconstructions were done using either Volocity 6.0.1 (PerkinElmer) or Fiji (https://imagej.net/ImageJ). All data presented in this study derive from observations made in at least 10 embryos/experiment in the case of fixed samples and on at least 3 embryos/experiment in the case of live imaging.

In order to quantify the number of Barhlla:GFP cells in the GCL, $14 \mu \mathrm{m}$ cryosections of $50 \mathrm{hpf}$ (barhlla:GFP;atoh7:gap43-RFP) double transgenic embryos were immunostained against GFP and Zn5 (also known as Alcama/ DM-GRASP/Neurolin/Zn8), a cell adhesion molecule that is transiently found on the entire surface of differentiated RGCs ${ }^{30}$. Barhlla:GFP positive cell nuclei were counterstained with DAPI. Sections were imaged with a Leica DM5000B compound microscope (20x objective). Three most central sections of each retina ( $\mathrm{n}=7$ retinae from 6 embryos) were imaged. Both GFP positive and GFP negative nuclei were counted manually in Volocity Analysis version 5.3 (Improvision). To exclude cells in the periphery, where the CMZ continues to give rise to all retinal cell classes, only cells within the central quadrant of the retina were counted as previously described ${ }^{20}$. For every retina, the percentage of GFP-positive cells was calculated as a mean of the proportions observed in each individual section.

Received: 20 February 2020; Accepted: 5 May 2020;

Published online: 01 June 2020

\section{References}

1. Juraver-Geslin, H. A., Ausseil, J. J., Wassef, M. \& Durand, B. C. Barhl2 limits growth of the diencephalic primordium through Caspase3 inhibition of beta-catenin activation. Proceedings of the National Academy of Sciences 108, 2288-2293 (2011).

2. Li, S., Qiu, F., Xu, A., Price, S. M. \& Xiang, M. Barhl1 regulates migration and survival of cerebellar granule cells by controlling expression of the neurotrophin-3 gene. J. Neurosci. 24, 3104-3114 (2004).

3. Bulfone, A. et al. Barhl1, a gene belonging to a new subfamily of mammalian homeobox genes, is expressed in migrating neurons of the CNS. Human Molecular Genetics 9, 1443-1452 (2000).

4. Colombo, A., Reig, G., Mione, M. \& Concha, M. L. Zebrafish BarH-like genes define discrete neural domains in the early embryo. Gene Expr. Patterns 6, 347-352 (2006).

5. Ding, Q., Joshi, P. S., Xie, Z.-H., Xiang, M. \& Gan, L. BARHL2 transcription factor regulates the ipsilateral/contralateral subtype divergence in postmitotic dI1 neurons of the developing spinal cord. Proceedings of the National Academy of Sciences 109, 1566-1571 (2012). 
6. Juraver-Geslin, H. A. \& Durand, B. C. Early development of the neural plate: new roles for apoptosis and for one of its main effectors caspase-3. Genesis 53, 203-224 (2015).

7. Reig, G., Cabrejos, M. E. \& Concha, M. L. Functions of BarH transcription factors during embryonic development. Developmental Biology 302, 367-375 (2007).

8. Barh, D. et al. BARHL1 Is Downregulated in Alzheimer's Disease and May Regulate Cognitive Functions through ESR1 and Multiple Pathways. Genes (Basel) 8 (2017).

9. Poschl, J. et al. Expression of BARHL1 in medulloblastoma is associated with prolonged survival in mice and humans. Oncogene 30, $4721-4730$ (2011).

10. Schuhmacher, L.-N., Albadri, S., Ramialison, M. \& Poggi, L. Evolutionary relationships and diversification of barhl genes within retinal cell lineages. BMC Evolutionary Biology 11, 340-17 (2011).

11. Patterson, K. D., Cleaver, O., Gerber, W. V., White, F. G. \& Krieg, P. A. Distinct expression patterns for two Xenopus Bar homeobox genes. Development Genes and Evolution 210, 140-144 (2000).

12. Saito, T., Sawamoto, K., Okano, H., Anderson, D. J. \& Mikoshiba, K. Mammalian BarH homologue is a potential regulator of neural bHLH genes. Developmental Biology 199, 216-225 (1998).

13. Poggi, L., Vottari, T., Barsacchi, G., Wittbrodt, J. \& Vignali, R. The homeobox gene Xbh1 cooperates with proneural genes to specify ganglion cell fate within the Xenopus neural retina. Development 131, 2305-2315 (2004).

14. Ding, Q. et al. BARHL2 Differentially Regulates the Development of Retinal Amacrine and Ganglion Neurons. Journal of Neuroscience 29, 3992-4003 (2009).

15. Mo, Z., Li, S., Yang, X. \& Xiang, M. Role of the Barhl2 homeobox gene in the specification of glycinergic amacrine cells. Development 131, 1607-1618 (2004).

16. Atac, D. et al. Atonal homolog 7 (ATOH7) loss-of-function mutations in predominant bilateral optic nerve hypoplasia. Human Molecular Genetics 29, 132-148 (2020).

17. Ghiasvand, N. M. et al. Deletion of a remote enhancer near ATOH7 disrupts retinal neurogenesis, causing NCRNA disease. Nature Neuroscience 14, 578-586 (2011).

18. Kay, J. N., Finger-Baier, K. C., Roeser, T., Staub, W. \& Baier, H. Retinal ganglion cell genesis requires lakritz, a zebrafish atonal homolog. Neuron 30, 725-736 (2001).

19. Prasov, L., Nagy, M., Rudolph, D. D. \& Glaser, T. Math5 (Atoh7) gene dosage limits retinal ganglion cell genesis. NeuroReport 23, 631-634 (2012).

20. Jusuf, P. R. et al. Biasing amacrine subtypes in the Atoh7 lineage through expression of Barhl2. J. Neurosci. 32, 13929-13944 (2012).

21. Jusuf, P., Harris, W. A. \& Poggi, L. Imaging retinal progenitor lineages in developing zebrafish embryos. Cold Spring Harb. Protoc. $2013(2013)$.

22. Jusuf, P., Harris, W. A. \& Poggi, L. Preparation of transgenic zebrafish embryos for imaging the developing retina. Cold Spring Harb. Protoc. 2013 (2013).

23. Dudczig, S., Currie, P. D., Poggi, L. \& Jusuf, P. R. In Vivo Imaging of Transgenic Gene Expression in Individual Retinal Progenitors in Chimeric Zebrafish Embryos to Study Cell Nonautonomous Influences. Journal of visualized experiments: JoVE. https://doi. org/10.3791/55490 (2017).

24. Scholpp, S., Poggi, L. \& Zigman, M. Brain on the stage - spotlight on nervous system development in zebrafish: EMBO practical course, KIT, Sept. 2013. Neural development 8, 23 (2013).

25. Poggi, L., Zolessi, F. R. \& Harris, W. A. Time-lapse analysis of retinal differentiation. Current Opinion in Cell Biology 17, 676-681 (2005).

26. Hu, M. \& Easter, S. S. Retinal neurogenesis: the formation of the initial central patch of postmitotic cells. Developmental Biology 207, 309-321 (1999).

27. Paolini, A. et al. Asymmetric inheritance of the apical domain and self-renewal of retinal ganglion cell progenitors depend on Anillin function. Development 142, 832-839 (2015).

28. Zolessi, F. R., Poggi, L., Wilkinson, C. J., Chien, C.-B. \& Harris, W. A. Polarization and orientation of retinal ganglion cells in vivo. Neural development 1, 2 (2006).

29. Poggi, L., Vitorino, M., Masai, I. \& Harris, W. A. Influences on neural lineage and mode of division in the zebrafish retina in vivo. J. Cell Biol. 171, 991-999 (2005).

30. Laessing, U. \& Stuermer, C. A. Spatiotemporal pattern of retinal ganglion cell differentiation revealed by the expression of neurolin in embryonic zebrafish. J. Neurobiol. 29, 65-74 (1996).

31. Jusuf, P. R. \& Harris, W. A. Ptf1a is expressed transiently in all types of amacrine cells in the embryonic zebrafish retina. Neural development 4, 34 (2009).

32. Jusuf, P. R. et al. Origin and Determination of Inhibitory Cell Lineages in the Vertebrate Retina. Journal of Neuroscience 31, 2549-2562 (2011).

33. Cepero Malo, M. et al. The Zebrafish Anillin-eGFP Reporter Marks Late Dividing Retinal Precursors and Stem Cells Entering Neuronal Lineages. PLoS ONE 12, e0170356 (2017).

34. Ding, Q., Balasubramanian, R., Zheng, D., Liang, G. \& Gan, L. Barhl2 Determines the Early Patterning of the Diencephalon by Regulating Shh. Mol. Neurobiol. 54, 4414-4420 (2017).

35. Staudt, N. \& Houart, C. The prethalamus is established during gastrulation and influences diencephalic regionalization. PLoS Biol. 5, e69 (2007).

36. Wilson, S. W., Ross, L. S., Parrett, T. \& Easter, S. S. J. The development of a simple scaffold of axon tracts in the brain of the embryonic zebrafish, Brachydanio rerio. Development 108, 121-145 (1990).

37. Chitnis, A. B., Patel, C. K., Kim, S. \& Kuwada, J. Y. A specific brain tract guides follower growth cones in two regions of the zebrafish brain. J. Neurobiol. 23, 845-854 (1992).

38. Chitnis, A. B. \& Kuwada, J. Y. Axonogenesis in the brain of zebrafish embryos. J. Neurosci. 10, 1892-1905 (1990).

39. Hjorth, J. \& Key, B. Development of axon pathways in the zebrafish central nervous system. Int. J. Dev. Biol. 46, 609-619 (2002).

40. Easter, S. S. J. \& Taylor, J. S. The development of the Xenopus retinofugal pathway: optic fibers join a pre-existing tract. Development 107, 553-573 (1989).

41. Ross, L. S., Parrett, T. \& Easter, S. S. J. Axonogenesis and morphogenesis in the embryonic zebrafish brain. J. Neurosci. 12, 467-482 (1992).

42. Tay, T. L., Ronneberger, O., Ryu, S., Nitschke, R. \& Driever, W. Comprehensive catecholaminergic projectome analysis reveals singleneuron integration of zebrafish ascending and descending dopaminergic systems. Nat Commun 2, 171 (2011).

43. Kastenhuber, E., Kratochwil, C. F., Ryu, S., Schweitzer, J. \& Driever, W. Genetic dissection of dopaminergic and noradrenergic contributions to catecholaminergic tracts in early larval zebrafish. J. Comp. Neurol. 518, 439-458 (2010).

44. Filippi, A. et al. Expression and function of $\mathrm{nr} 4 \mathrm{a} 2, \operatorname{lmx} 1 \mathrm{~b}$, and pitx3 in zebrafish dopaminergic and noradrenergic neuronal development. BMC Dev Biol 7, 135 (2007).

45. Randlett, O., Poggi, L., Zolessi, F. R. \& Harris, W. A. The oriented emergence of axons from retinal ganglion cells is directed by laminin contact in vivo. Neuron 70, 266-280 (2011).

46. Norden, C. Pseudostratified epithelia - cell biology, diversity and roles in organ formation at a glance. Journal of Cell Science 130, 1859-1863 (2017).

47. Schmidt, R., Strähle, U. \& Scholpp, S. Neurogenesis in zebrafish - from embryo to adult. Neural development 8, 3 (2013). 
48. Lyons, D. A., Guy, A. T. \& Clarke, J. D. W. Monitoring neural progenitor fate through multiple rounds of division in an intact vertebrate brain. Development 130, 3427-3436 (2003).

49. Juan, G. et al. Histone $\mathrm{H} 3$ phosphorylation and expression of cyclins A and B1 measured in individual cells during their progression through G2 and mitosis. Cytometry 32, 71-77 (1998).

50. Bernardos, R. L. \& Raymond, P. A. GFAP transgenic zebrafish. Gene Expr. Patterns 6, 1007-1013 (2006).

51. Wilson, S. W. \& Easter, S. S. J. A pioneering growth cone in the embryonic zebrafish brain. Proceedings of the National Academy of Sciences 88, 2293-2296 (1991).

52. Pittman, A. J., Law, M. Y. \& Chien, C. B. Pathfinding in a large vertebrate axon tract: isotypic interactions guide retinotectal axons at multiple choice points. Development 135, 2865-2871 (2008).

53. Li, S. \& Xiang, M. Barhll is required for maintenance of a large population of neurons in the zonal layer of the superior colliculus. Dev. Dyn. 235, 2260-2265 (2006)

54. Amamoto, R. \& Arlotta, P. Development-inspired reprogramming of the mammalian central nervous system. Science 343, 1239882 (2014).

55. Kimmel, C. B., Ballard, W. W., Kimmel, S. R., Ullmann, B. \& Schilling, T. F. Stages of embryonic development of the zebrafish. Dev. Dyn. 203, 253-310 (1995).

56. McNabb, A., Scott, K., Ochsenstein, E. V., Seufert, K. \& Carl, M. Don't be afraid to set up your fish facility. Zebrafish 9, 120-125 (2012).

57. Liu, P., Jenkins, N. A. \& Copeland, N. G. A highly efficient recombineering-based method for generating conditional knockout mutations. Genome Research 13, 476-484 (2003).

58. Auer, T. O., Duroure, K., Concordet, J.-P. \& Del Bene, F. CRISPR/Cas9-mediated conversion of eGFP- into Gal4-transgenic lines in zebrafish. Nat Protoc 9, 2823-2840 (2014).

59. Bussmann, J. \& Schulte-Merker, S. Rapid BAC selection for tol2-mediated transgenesis in zebrafish. Development 138, 4327-4332 (2011).

60. Suster, M. L., Abe, G., Schouw, A. \& Kawakami, K. Transposon-mediated BAC transgenesis in zebrafish. Nat Protoc 6, 1998-2021 (2011).

61. Balciunas, D. et al. Harnessing a high cargo-capacity transposon for genetic applications in vertebrates. PLoS Genet 2, e169 (2006).

62. Piatkevich, K. D., Malashkevich, V. N., Almo, S. C. \& Verkhusha, V. V. Engineering ESPT pathways based on structural analysis of LSSmKate red fluorescent proteins with large Stokes shift. J. Am. Chem. Soc. 132, 10762-10770 (2010).

63. Dona, E. et al. Directional tissue migration through a self-generated chemokine gradient. Nature 503, 285-289 (2013).

64. Armant, O. et al. Genome-wide, whole mount in situ analysis of transcriptional regulators in zebrafish embryos. Developmental Biology 380, 351-362 (2013).

\section{Acknowledgements}

We thank J. Wittbrodt and K. Lust for kindly providing the gfap:lss-kate DNA construct made by K. Lust in the laboratory of J.Wittbrodt. We thank B. Wittbrodt, E. Leist, A. Saraceno, M. Majewski, B. Seiferling, T. Kellner at COS, University of Heidelberg, and I. Mazzeo and S. Robbiati at CIBIO, University of Trento, for fish maintenance and technical assistance. We are also thankful for the support of the core imaging facility at CIBIO, University of Trento. S.A. was supported by the Landesgraduiertenförderung (Funding program of the State of Baden Württemberg, Germany). This work was supported by Deutsche Forschungsgemeinschaft Research Grant PO 1440/1-1 to L.P and by University of Trento.

\section{Author contributions}

L.P. conceptualized, supervised the project and wrote the manuscript. S.A. performed the research, prepared the figures and wrote the manuscript together with L.P. M.C., F.D.B. and U.S. commented and proofed the manuscript. M.C. contributed with the conceptualization of the manuscript. S.A. generated the barhl1.2:gal4-vp16 BAC construct in the laboratory of F.D.B to perform mosaic expression experiments. O.A. and U.S. made the $\operatorname{Tg}($ barhl1a:GFP) transgenic line and O.A. performed in situ analysis of barhl1a and $l m x 1 b 1$. T.A.G. performed Cxcr4b immuno-histo labelling and quantification of Barhlla:GFP cells under the supervision of S.A. and L.P. All authors revised and approved the final version of the manuscript.

\section{Competing interests}

The authors declare no competing interests.

\section{Additional information}

Supplementary information is available for this paper at https://doi.org/10.1038/s41598-020-65435-w.

Correspondence and requests for materials should be addressed to L.P.

Reprints and permissions information is available at www.nature.com/reprints.

Publisher's note Springer Nature remains neutral with regard to jurisdictional claims in published maps and institutional affiliations.

Open Access This article is licensed under a Creative Commons Attribution 4.0 International License, which permits use, sharing, adaptation, distribution and reproduction in any medium or format, as long as you give appropriate credit to the original author(s) and the source, provide a link to the Creative Commons license, and indicate if changes were made. The images or other third party material in this article are included in the article's Creative Commons license, unless indicated otherwise in a credit line to the material. If material is not included in the article's Creative Commons license and your intended use is not permitted by statutory regulation or exceeds the permitted use, you will need to obtain permission directly from the copyright holder. To view a copy of this license, visit http://creativecommons.org/licenses/by/4.0/.

(C) The Author(s) 2020 